\title{
CFD prediction of air flow past a full helicopter configuration
}

\author{
Massimo Biava ${ }^{\mathrm{a}}$, Walid Khier ${ }^{\mathrm{b}}$, Luigi Vigevano ${ }^{\mathrm{c}, *}$ \\ a AgustaWestland-Politecnico Advanced Rotorcraft Center S.r.I. (AWPARC), Via G. Durando 10, 20158 Milan, Italy \\ ${ }^{\mathrm{b}}$ Institut für Aerodynamik und Strömungstechnik, Deutsches Zentrum für Luft- und Raumfahrt e.V. (DLR), Lilienthalplatz 7, 38108 Braunschweig, Germany \\ c Dipartimento di Ingegneria Aerospaziale, Politecnico di Milano, Via La Masa 34, 20156 Milan, Italy
}

\section{A R T I C L E I N F O}

Article history:

Available online $\mathrm{xxxx}$

\section{Keywords:}

Complete helicopter flow field

Unsteady RANS

Fluid-structure coupling

\begin{abstract}
A B S T R A C T
The present study is one of the first attempts to exploit the GOAHEAD data base to perform a code-tocode evaluation on complete helicopter aerodynamics. The numerical results of two GOAHEAD partners, the German Aerospace Center (DLR) and Politecnico di Milano (PoliMi) are presented and compared to experimental measurements. The study also addresses an evaluation of two different approaches to predict helicopter flows. The first, applied by DLR, accounts for rotor trim and elastic effects by weak fluid-structure coupling. The PoliMi approach, on the other hand, enforces a prescribed kinematics, taken directly from the experiment, on a rigid blade. The simulations refer to a complete helicopter windtunnel model, featuring a scaled NH90 fuselage, the ONERA 7AD main rotor, a scaled BO105 tail rotor, a rotor hub and a pylon, all located inside the $8 \mathrm{~m} \times 6 \mathrm{~m}$ test section of the DNW low-speed wind tunnel. The flight conditions correspond to cruise flight at $\mathrm{Ma}=0.204$ and fuselage attitude $\alpha=-2.5^{\circ}$. The comparisons demonstrate the capability of present unsteady RANS solvers to predict flow fields around complete helicopters.
\end{abstract}

(c) 2011 Elsevier Masson SAS. All rights reserved.

\section{Introduction}

Computational Fluid Dynamics (CFD) has evolved as a prediction tool in the helicopter research and development field over the past two decades. Application of CFD to the simulation of viscous flow over individual components of the aircraft, such as the fuselage and the rotor, was carried out successfully [30], demonstrating the usefulness of CFD in the early design phase of the aircraft.

To consider the complete rotor-fuselage configuration has been however much more challenging, because of: i) the geometrical complexity and the resulting grid requirements, both in terms of dimensions and generation effort; ii) the need to handle the relative motion between rotor blades and fuselage, thus requiring sophisticated numerical techniques, like the overlapping grid Chimera method [1] or the sliding mesh approach [32].

For these reasons early attempts focused on either the simulation of a realistic fuselage geometry coupled with a steady actuator disk model of the rotor (see [27] for a code-to-code comparison), or the simulation of a fully unsteady rotor flow over a simplified shape fuselage, like the Georgia Tech experiment [19] or the ROBIN test case $[7,15,33]$.

Simulations of the viscous, fully unsteady flow over a complete helicopter configuration of industrial relevance have been carried

\footnotetext{
* Corresponding author. Tel.: +39 0223998317; fax: +39 23998334.

E-mail addresses: walid.khier@dlr.de (W. Khier), luigi.vigevano@polimi.it (L. Vigevano).
}

out only very recently. Renaud et al. [26] presented a comparison with experimental pressure measurements for a Dauphin $365 \mathrm{~N}$ model in which the fenestron tail rotor was not included. In the same publication the NH90 helicopter, with both main and tail rotors, was also simulated, although in this latter case no experimental validation was possible. The BO105 complete configuration at wind-tunnel model scale has been simulated in [18], where a limited comparison with experimental unsteady pressures over the fuselage and both rotors was presented. These early attempts of considering the complete helicopter were carried out using multiblock, structured grids and the Chimera method, with rigid blades and prescribed rotor kinematics. A noteworthy comparison of different computational methodologies using prescribed kinematics is reported in [24], where overset grid and unstructured grid solutions were evaluated.

One of the reasons that prevented a careful validation of the present helicopter CFD capability has been the lack of a suitable experimental database. This motivated the launch in 2005 , by a consortium of leading research institutes and helicopter manufacturers, of the EU project GOAHEAD [22]. The main objectives of the project were to create an experimental database for the validation of CFD codes in helicopter related applications, and to apply this database to validate modern CFD tools. The measurements were successfully carried out at the German-Dutch low speed wind tunnel (DNW) in spring 2008. The GOAHEAD test campaign included detailed steady and unsteady surface pressure measurements on the helicopter fuselage and rotors, transition locations, wind-tunnel inflow velocity and turbulence kinetic energy levels 
and PIV velocity field measurements for a wide array of flight and rotor loading conditions. A code validation activity was carried out separately within the project. The blind validation computations from several partners were briefly documented in [6].

Elastic deformation of loaded helicopter main rotor blades is undoubtedly a major factor affecting the performance of the rotor. Torsional, flapping and lead-lag oscillations alter the direction of the flow relative to the blade, leading to a deviation in rotor forces with respect to its performance under rigid blade assumption [23]. There have been two main approaches to include blade elasticity: weak (or loose) coupling or strong coupling with a flight and structural mechanics tool; both options increase the complexity and computational costs considerably. Nevertheless, the most recent advances in helicopter CFD account for complete helicopter configurations with weak CSD/CFD coupling. In [4] the two-rotor $\mathrm{CH}-$ 47 helicopter was considered, while the GOAHEAD wind-tunnel model was simulated in $[12,17]$. A preliminary comparison of the trimmed blind calculations for the GOAHEAD model with experiments was presented in [16].

Obviously, the significance of fluid-structure coupling increases as the stiffness of the blades decreases. For moderately loaded stiff blades, as those employed in model rotor tests, it is not clear how far elastic effects may affect the accuracy of the numerical predictions, and whether these differences justify the additional computational overhead. This consideration motivated the code-tocode comparison reported in the present work. The contributions of DLR and PoliMi to the post-test validation activity of GOAHEAD are reported. The numerical predictions are evaluated against the GOAHEAD experimental database to assess the ability and accuracy of DLR and PoliMi URANS solvers to predict the complex flow phenomena related to helicopters. Both codes rely on similar overset grid methods, but differ in the treatment of the rotor blade kinematics: while a weak fluid-structure coupling to trim the rotor is applied by DLR, in PoliMi approach the blade motion is directly taken from the experiment and the blade are considered rigid. In this way, the effect of elastic deformation on the stiff ONERA 7AD blade may be assessed by comparison of the two different simulation approaches.

The numerical methods are briefly described in Section 2, while some details on the simulated helicopter model, computational grids and flow conditions are given in Section 3. Section 4 is dedicated to the numerical results and their comparison with the experimental data. Finally, the conclusions are listed in the fifth and last section of the paper.

\section{Numerical methods}

The numerical simulations presented and discussed hereinafter are based on the time-accurate solution of the Reynolds (Favre) averaged Navier-Stokes equations in three dimensions by means of two CFD block-structured, finite volume codes: FLOWer [20,21] by DLR and ROSITA [5] by PoliMi.

Several features of the numerical methods employed in the present study are similar among the two solvers: cell-centered finite volume spatial discretization on multi-block structured grids, formulated as to account for moving and deforming meshes satisfying the geometry conservation law, central discretization of the viscous fluxes, implicit time integration using the dual-time stepping method, moving Chimera technique to facilitate the grid generation process and represent the motion of the blades in the simulation, characteristic-type boundary conditions, parallelization making use of the MPI framework.

There are however some noticeable differences in the presented simulations, regarding the spatial discretization of the convective fluxes, the turbulence model, the details of the adopted Chimera algorithm and the fluid-structure coupling.

\subsection{Convective fluxes}

In the present FLOWer simulations, the convective fluxes are discretized with second order central differences. Third order numerical dissipation is added to the convective fluxes to ensure numerical stability. These dissipative contributions are reduced to first order when a shock is detected. Smooth transition from the third to the first order is realized by linear combination of both terms.

The ROSITA solver makes use of the Roe's scheme. Second order accuracy is obtained through the use of MUSCL extrapolation supplemented with a modified version of the Van Albada limiter introduced by Venkatakrishnan [34].

\subsection{Turbulence model}

FLOWer contains a large array of statistical turbulence models, ranging from algebraic and one-equation eddy viscosity models [28] to seven-equation Reynolds stress model. In this work a slightly modified version of Wilcox's two-equation $k-\omega$ model is used [35]. Unlike the main flow equations, Roe's scheme is employed to compute the turbulent convective fluxes.

The one-equation Spalart-Allmaras model [31] is used in the ROSITA simulations.

\subsection{Chimera algorithms}

The implementation of the Chimera approach in FLOWer follows the ideas of Benek [2]. Theoretically, an unlimited number (up to the code dimension limits) of hierarchies of relative motions can be specified in time, and applied to the different elements of the geometry. Each level of the hierarchy defines a separate reference frame in which motions can be specified independently of the inertial frame of reference, thus allowing any combination of translation and rotation motions to be realized by a series of simple co-ordinate transformations. The search for cells, required for interpolation, is performed by an Alternating Digital Tree (ADT) search method. The hole cutting procedure does not imply any hierarchical mesh dependencies: to mark points being inside a solid body, a simple auxiliary grid which encloses the solid body must be provided by the user. All points of the grid inside the auxiliary grid are excluded from the flow calculation. This leaves uncontrolled the extent of the overlapping regions within the domain. Special corrections are applied to overlapping regions located close to solid walls [29].

PoliMi's approach has a similar generality about the relative motion of the solid bodies, but follows a different approach for hole cutting and tagging, which is derived from that originally proposed by Chesshire and Henshaw [9], with modifications to improve robustness and performance. The tagging procedure accounts for a hierarchical grid ordering and attempts to minimize the overlap regions. To speed up the search of donor points, both oct-tree and ADT data structures are considered.

Both codes employ non-conservative tri-linear interpolation to transfer information among the different grids. For integration of the aerodynamic forces on overlapping surface grids, a special treatment proposed by Chan and Buning [8] is used.

\subsection{Fluid-structure coupling}

This feature represents the main difference in the simulation approaches used in the present study. While the ROSITA computations are run with a rigid blade prescribed kinematics, measured during the experimental tests, the FLOWer results are gathered using a weak CSD/CFD coupling. 

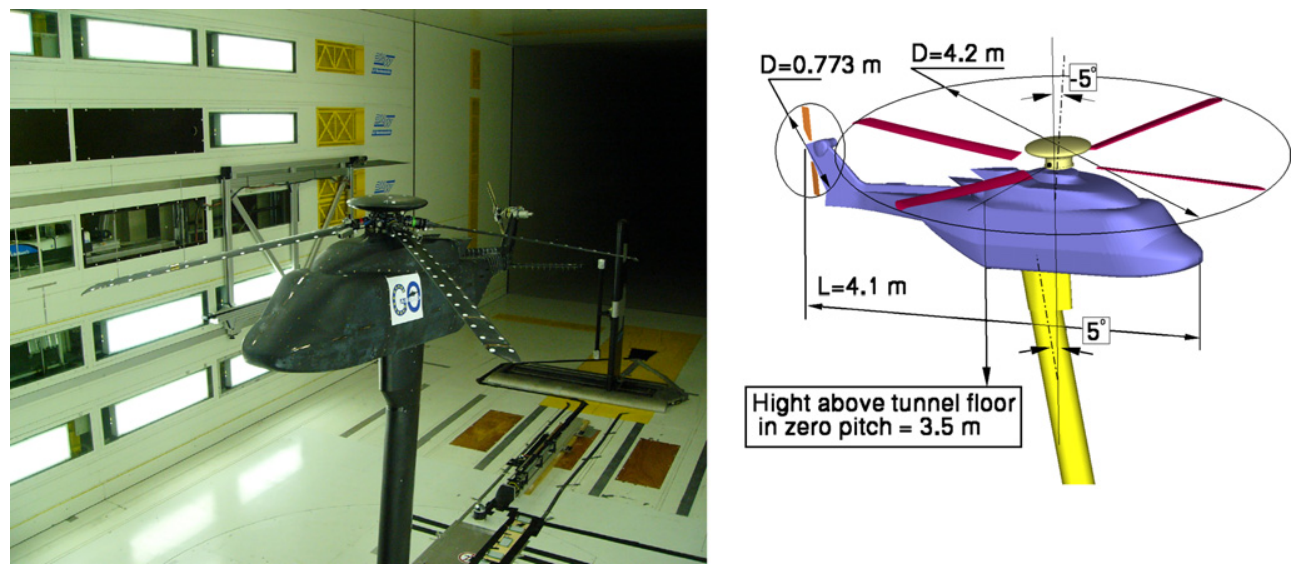

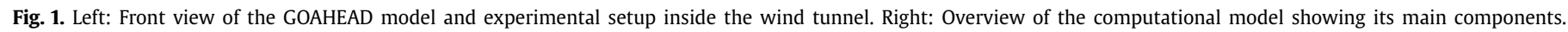
Wind tunnel section not shown.
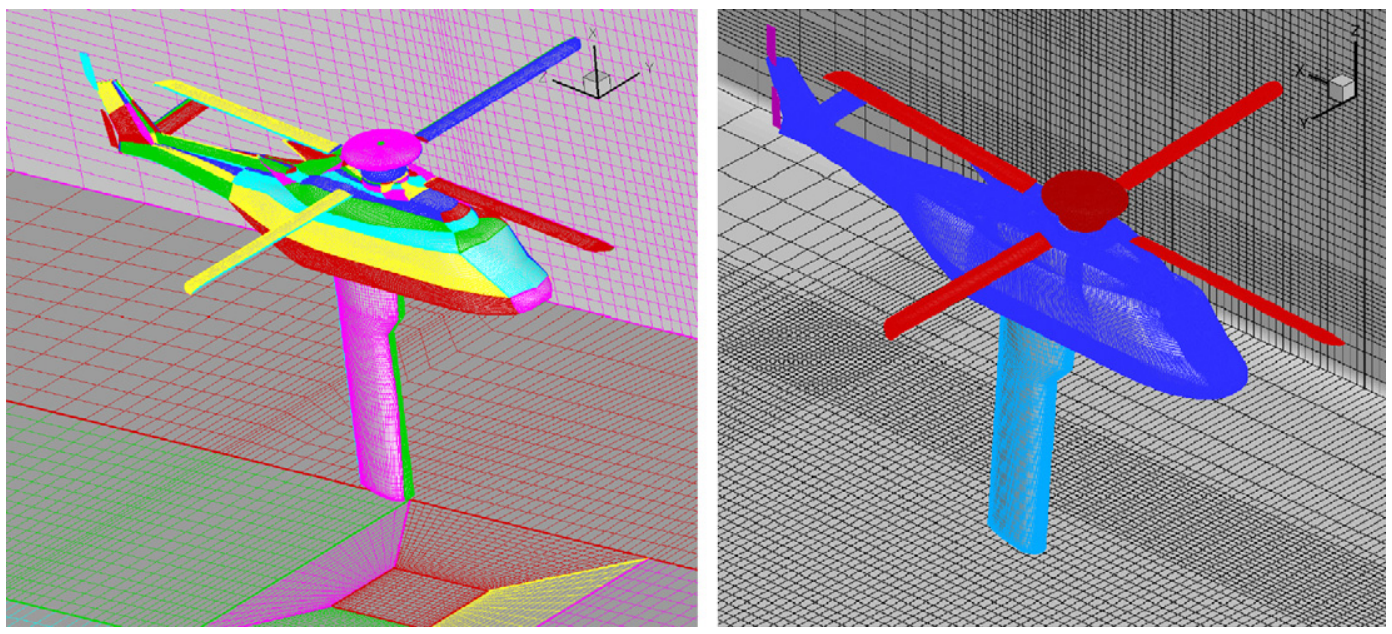

Fig. 2. Surface grid on the model and wind tunnel walls. Left: DLR grid. Right: PoliMi grid.

In the DLR computations, the rotor was trimmed using the stand alone flight mechanics tool HOST (Helicopter Overall Simulation Tool) [3] to generate the experimental weight, lateral and propulsive force coefficients. The resulting rotor controls and elastic deformation were used to modify the blade surface geometry following the approach presented in $[10,11,13]$. The process, described in detail in [12,17], is repeated until the variations in elastic blade deformation and rotor control angles have fallen below a user defined tolerance.

\section{Computational details and experimental conditions}

The GOAHEAD helicopter model considered in the present work consists of a $4.1 \mathrm{~m}$ long NH90 fuselage, the ONERA 7AD main rotor, with blade radius of $2.1 \mathrm{~m}$, a reduced scale BO105 tail rotor and a main rotor hub, which is simplified to a cylindrical element and an elliptical fairing. Both main and tail rotors are represented by isolated blades. The tail rotor hub is not included in the computational model.

The fuselage shape reproduces the complex geometrical details of the engine exhausts. It corresponds to the actual wind-tunnel model geometry which has been carefully measured before the test campaign.

The simulations consider the helicopter model installed within the $8 \mathrm{~m} \times 6 \mathrm{~m}, 20 \mathrm{~m}$ long test section of the DNW wind tunnel, as shown in Fig. 1. The model is mounted on a faired support. It should be noted that the main rotor rotates in clockwise direction
Table 1

Summary of DLR and PoliMi Grid dimensions.

\begin{tabular}{lcc}
\hline No. of points $\left(\times 10^{6}\right)$ & PoliMi & DLR \\
\hline Fuselage & 17.4 & 18.1 \\
Main rotor blade $(\times 4)$ & 1 & 0.87 \\
Tail rotor blade $(\times 2)$ & 0.5 & 0.35 \\
Rotor hub & 2 & 2.12 \\
Strut & 1.3 & 0.9 \\
Wind tunnel & 1.3 & 0.3 \\
Total (Mil. point) & 27 & 25.6 \\
\hline
\end{tabular}

as seen from above. The upper vertical tail rotor blade is advancing and the lower vertical blade is retreating.

Among the several flight conditions reproduced during the GOAHED tests, this work consider the cruise/tail shake condition that corresponds to a forward flight at Mach number $M=0.204$, advance ratio $\mu=0.33$ with $-2.5^{\circ}$ fuselage pitch angle. Careful measurements of the inflow characteristics have been carried out during the tests to achieve well defined boundary conditions for the simulations [25].

\subsection{Numerical grids}

Multi-block grids around the different elements were subdivided into 10 Chimera components: fuselage, rotor hub, four main rotor blades, two tail rotor blades, model strut and wind tunnel walls. Fig. 2 shows the surface grid for the complete helicopter configuration, while Table 1 lists the major characteristics of the numerical grids used. 

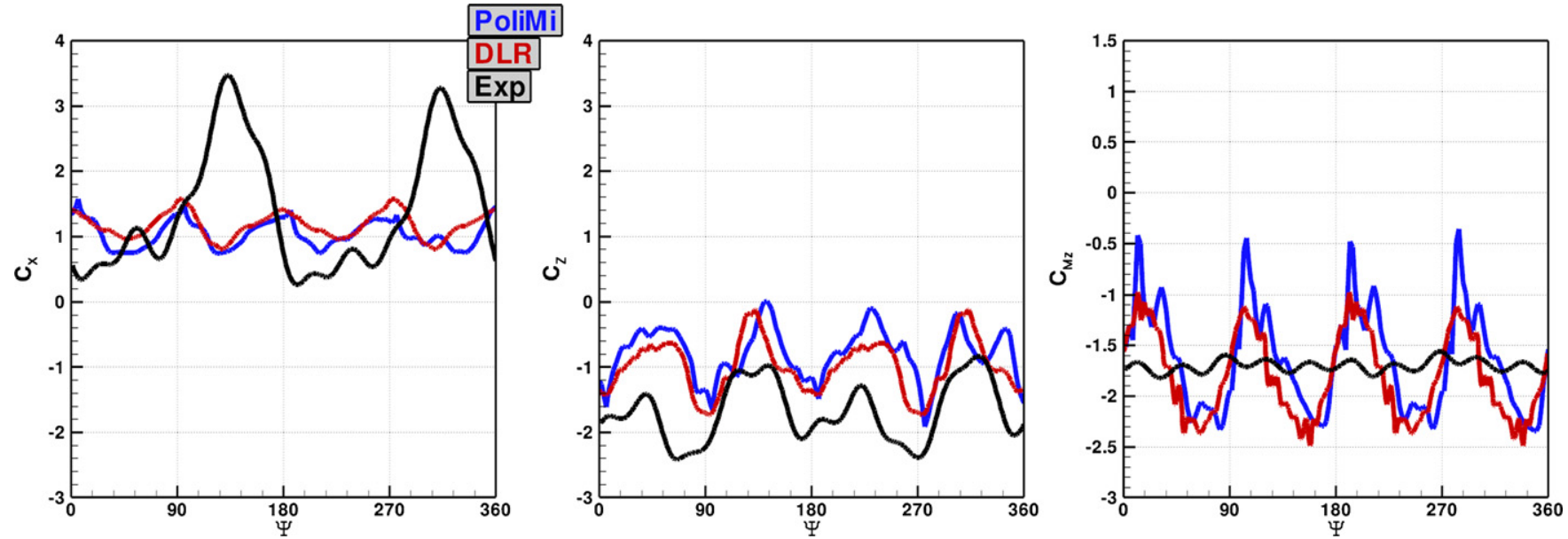

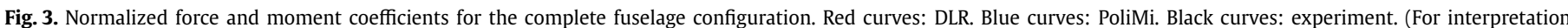
of the references to color in this figure, the reader is referred to the web version of this article.)
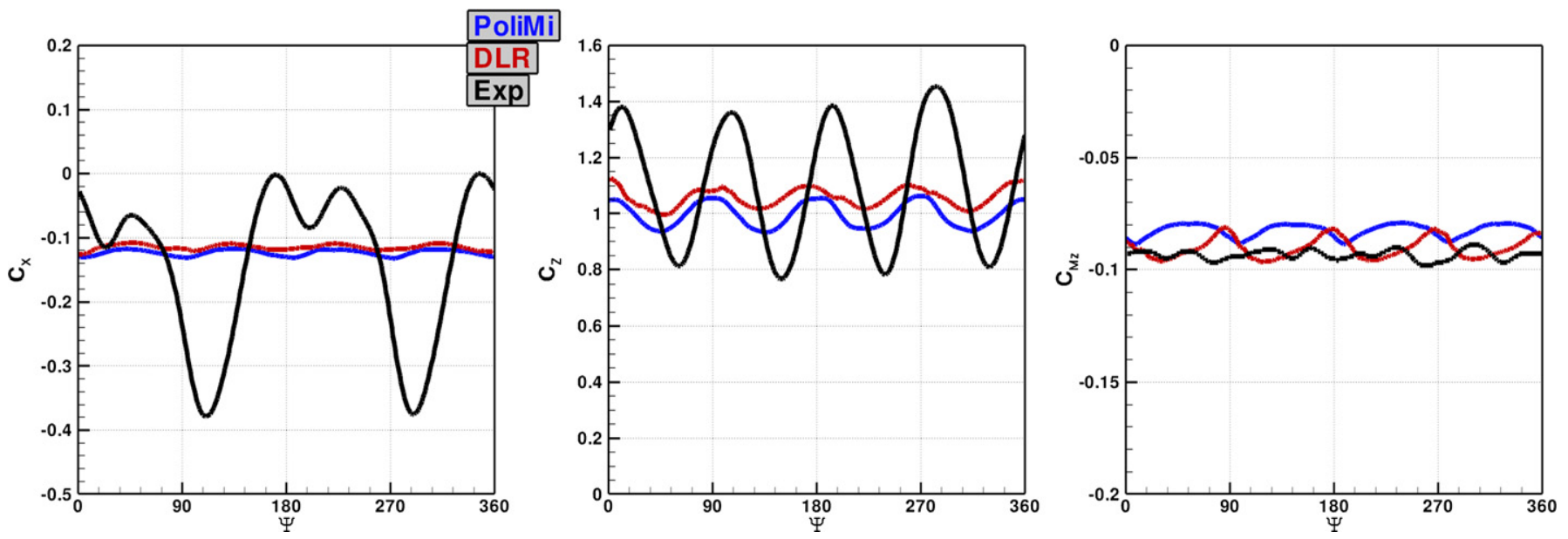

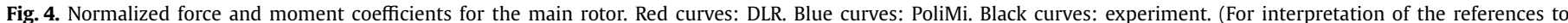
color in this figure, the reader is referred to the web version of this article.)

Table 2

Summary of DLR and PoliMi computational parameters.

\begin{tabular}{lll}
\hline & PoliMi & DLR \\
\hline Azimuthal step $\left(^{\circ}\right)$ & 1 & $1-2$ \\
N. of time steps in pseudo-time & 50 & $50-100$ \\
CFL number & 4 & 5.5 \\
CSD/CFD iterations & 0 & 4 \\
N. of main rotor revolutions & 4 & 5 \\
N. of processors & 240 & 8 \\
Type of processor & Xeon 5660 & NEC-SX8 \\
Total memory requirement $(\mathrm{GB})$ & 25 & 30 \\
CPU-time per revolution $(\mathrm{h})$ & 47 & 80 \\
\hline
\end{tabular}

\subsection{Computational details}

The most relevant computational details for the two solvers are summarized in Table 2. In the coupled DLR simulation some parameters were modified during the iterative process: for instance the azimuthal step was kept at $2^{\circ}$ for the initial phase of the iteration and then modified to $1^{\circ}$. The number of time steps employed in pseudo-time corresponds to a reduction of the residual of 2-3 orders of magnitude.

\section{Results}

Due to the complexity of the unsteady flow around the complete helicopter model, the analysis and comparison of the results is not a trivial task. We will then proceed towards a direction of greater detail, going from the examination of the global loads down to the investigation of some features of the flow field in localized regions.

\subsection{Global loads}

Global force and moment coefficients are reported in normalized form, due to contractual obligations. The actual coefficients are scaled with arbitrary reference values. The coefficients refer to wind tunnel, i.e. wind axes, for both fuselage and main rotor loads. Measurements are phase averaged over 32 rotor revolutions.

Normalized coefficients for the complete fuselage configuration (cabin, engine casing, tail boom, tail fin, horizontal stabilizer and rotor head) are shown in Fig. 3. Both simulations yield very similar results, with some noticeable differences with the experimental data. The experimental drag coefficient is characterized, in addition to a $4 / \mathrm{rev}$ frequency, by a $2 / \mathrm{rev}$ occurrence, with peaks at $\Psi=130^{\circ}$ and $\Psi=310^{\circ}$, that is not represented by the numerical results. This occurrence seems due to the interaction with the main rotor, which propulsive force (see Fig. 4a) shows a similar 2/rev component with a $20^{\circ}$ phase advancement. The negative lift of this configuration is slightly underestimated by both simulations. The numerical yawing moment results are dominated by the 4/rev influence of the main rotor, while the experimental signal shows mainly the effect of the tail rotor: the rotation speed of the twoblade tail rotor is fivefold the main rotor rotation speed, so that the tail rotor characteristic frequency is $10 /$ rev. 


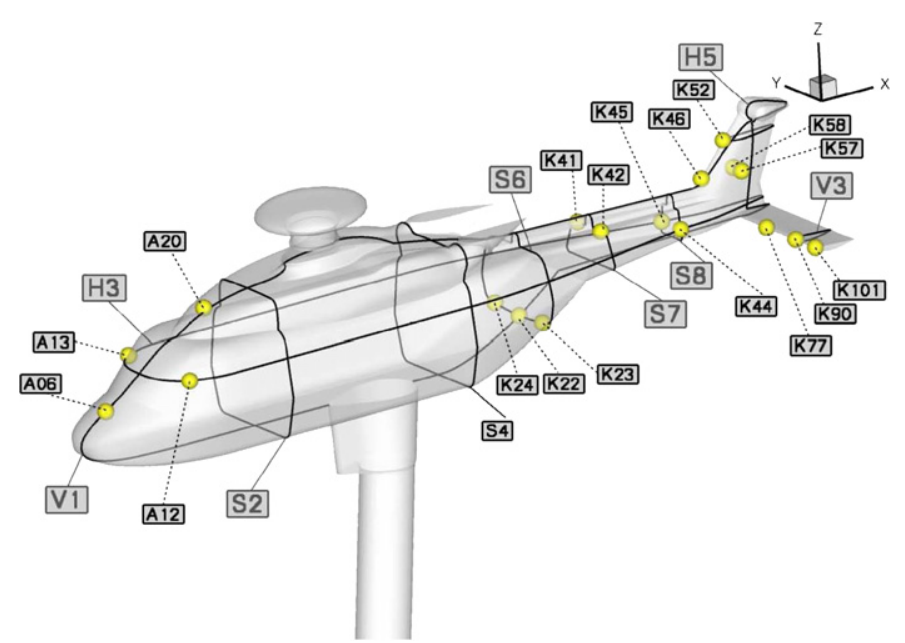

Fig. 5. Location of the fuselage cross-sectional planes and unsteady sensor positions used for comparison amongst CFD and experiment.

Normalized loads for the main rotor are reported in Fig. 4. Also in this case the two codes achieve very similar results. The thrust level is underestimated, with respect to the experimental values. However the agreement for torque is fairly good. Quantitatively, the calculated main rotor power reaches $99.7 \%$ of the experimental value for the DLR results, while only $85 \%$ for PoliMi results, thus indicating that the rigid blade assumption underestimates power consumption. Fluid-structure coupling considerably improves the power prediction with an error of $0.3 \%$ only.

\subsection{Pressure on fuselage}

The fuselage is equipped with a total number of 130 unsteady pressure sensors. The location of some selected sensors is depicted in Fig. 5. Strong oscillations were found in the experimental pressures [16], which in some cases are probably due to vibration of the model inside the tunnel. The experimental data shown in the forthcoming figures were obtained by averaging the pressure signals recorded for each azimuthal position over 130 revolutions.

One preliminary observation needs to be done on the comparison of the pressure time-histories on the fuselage. The simulations reproduce carefully the actual wind-tunnel model geometry, as measured. However, the actual location of the pressure sensors on the model has not been measured during the test. The only available information was the sensor location as defined on the nominal model geometry, that used for the blind-test calculations. Since actual and nominal geometries of the fuselage do not match precisely, the extraction of the numerical results in correspondence of the single sensor location has to be considered only approximate, especially for those sensors that are placed in regions of the body surface where strong spatial gradients are present, like the fin leading edge for instance. This inaccuracy is reduced when comparing pressure sectional distributions. Furthermore, such a problem is not encountered for the blade pressure sensors, since their position is known accurately.

Fig. 6 compares the computed pressure signals with the experiment for 12 selected sensors. Broadly good agreement between measurements and computations can be observed for the sensors on the nose (Fig. 6a) and on the windscreen (Fig. 6b and 6c) regions. The influence of the rotor is well captured in the computations in terms of frequency and phase. Note how the overpressure due to the blade passage is larger at the upwind side of the windscreen (Fig. 6b), leading to an asymmetry of the pressure distribution. PoliMi results show slight underestimation of pressure on the nose and the advancing blade side. Both sets of CFD results predict higher pressure values on the retreating side (Fig. 6c).

The pressure signals on the upper side of the tail boom, in Fig. $6 \mathrm{~d}$ and $6 \mathrm{e}$, are still characterized by a strong influence of the blade passage, i.e. feature a dominant $4 /$ rev frequency, although some higher frequencies are observed since the sensors are located within the wake of the hub fairing. The raw experimental data present however much higher oscillations, which are damped out during the averaging process over the 130 revolutions. These high frequency oscillations can be observed also in the numerical results, especially on the advancing blade side. The average value over one revolution is in fact well predicted by both codes. High frequency oscillations are observed in the computations also on the lower side of the tail boom (Fig. $6 \mathrm{f}$ and $6 \mathrm{~g}$ ). PoliMi results are closer to the experiment on the advancing side as shown in Fig. $6 \mathrm{f}$ but with strong overshoots of the peak values, while DLR results follow the experimental trend but at a nearly constant offset. As far as the average values are concerned, the agreement becomes better on the retreating side (Fig. $6 g$ ).

The effect of the main rotor cannot be easily identified in the tail fin signals depicted in Fig. 6h and 6i, due to strong interference between the fuselage and main rotor wakes with the tail rotor and tail fin. DLR results show however an evident $4 /$ rev pattern. PoliMi data is dominated by high frequency oscillation but remains close to the measurements. It has to be noticed that for these sensors, close to the fin leading edge, the approximation of the extraction of the numerical results is the largest.

Fig. $6 j-1$ contains the pressure data on the advancing side (Fig. 6j), symmetry plane (Fig. 6k) and the retreating side (Fig. 6l) of the back door. Inspection of the measured data reveals slight decrease in average pressure from the advancing side towards the retreating side (Fig. $6 \mathrm{j}$ to $\mathrm{l}$ ). Pressure pulses are observed on the advancing side sensor indicating influence of the rotor on the flow in this region. The amplitude of the suction becomes almost uniform as the symmetry plane is approached and increases slightly on the retreating side. A similar behavior cannot be observed in the numerical data. DLR and PoliMi predictions overestimate the pressure level and do not show any harmonic evolution with the azimuth angle, but the influence of a turbulent wake.

Snapshots of surface pressure distributions along the fuselage symmetry plane at several azimuth angles are illustrated in Fig. 7. Accurate prediction of the rapid pressure drop on the leading edge of the fuselage and the subsequent pressure recovery downstream the mast fairing can be clearly seen in the figure. DLR and PoliMi results show similar level of accuracy except in the nose and wind shield areas where the DLR results are slightly closer to the experimental data. The pressure recovery zone downstream the mast fairing is another area where differences in the numerical data can be seen. The differences between the numerical results are however small.

In summary, we can conclude that both codes feature a fairly good agreement, among themselves and with the experiments, on the unsteady pressure distribution at the front part of the fuselage. The comparison of the unsteady pressure signals in the aft parts of the fuselage (tail boom, fin, back door), were a complex turbulent wake take place, is less satisfactory, but it should be remembered that the reported experimental data are averaged over 130 rotor revolutions while the computed results are not. The differences among DLR and PoliMi results - obtained with different turbulent models - induce to put unsteady turbulence models under scrutiny.

\subsection{Pressure on rotors}

Computed and measured pressure coefficients on the main rotor blade are compared in Figs. 8 to 10 . The figures respectively 
a)

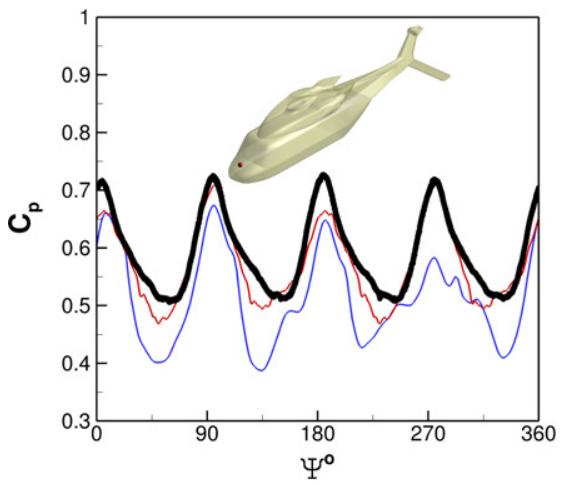

d)

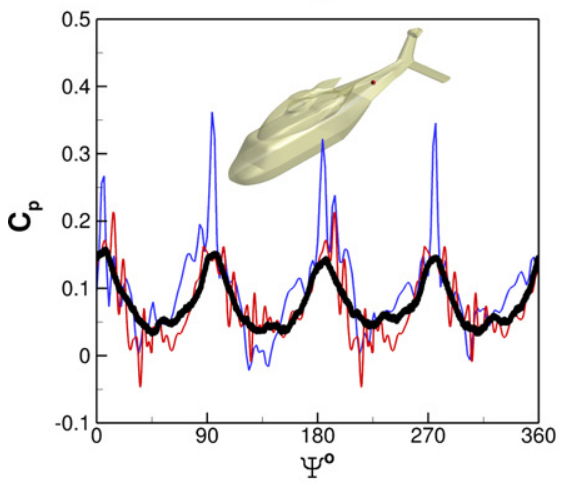

g)

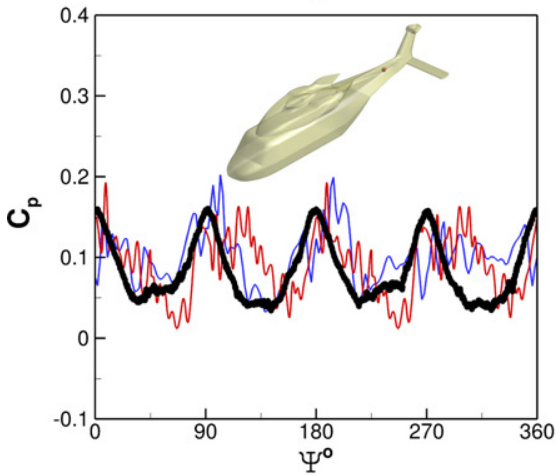

j)

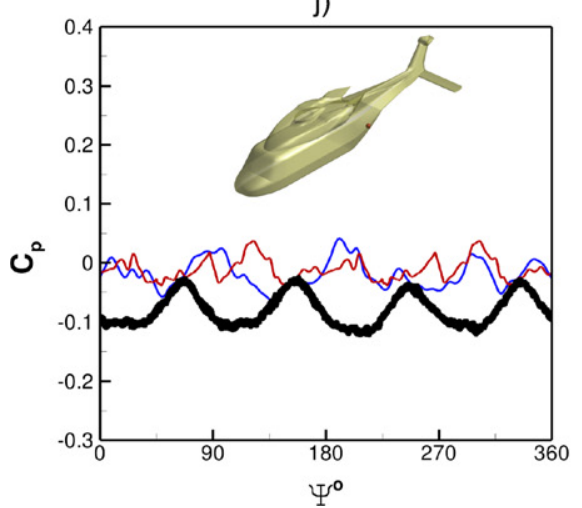

b)

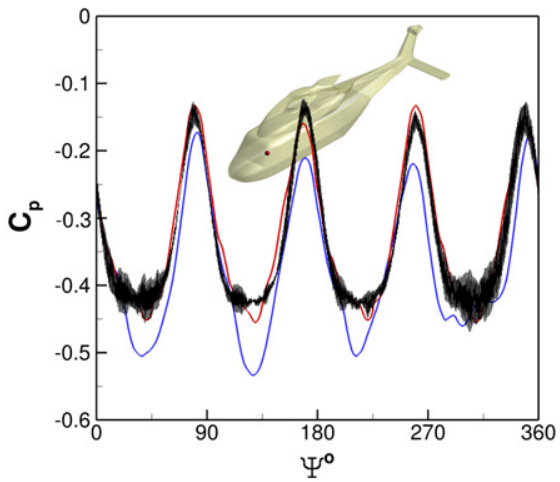

e)

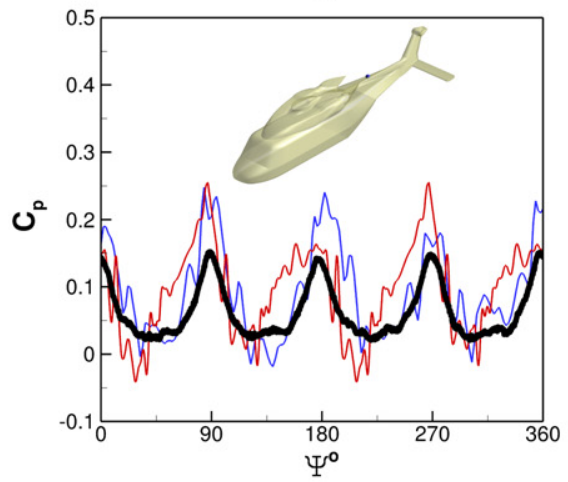

h)

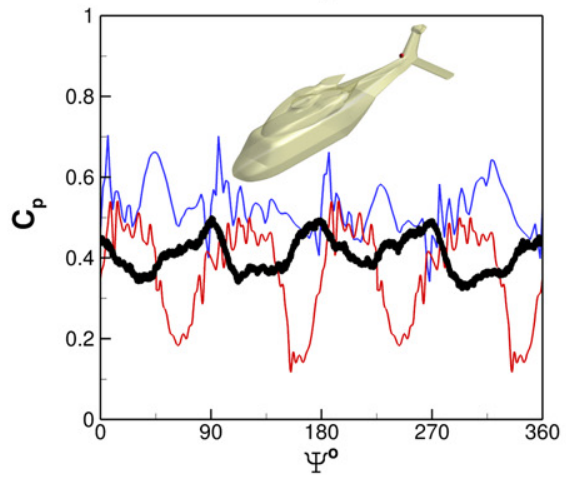

k)

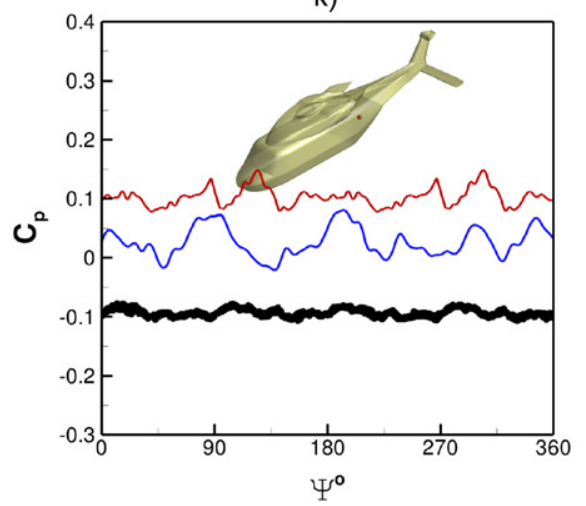

c)

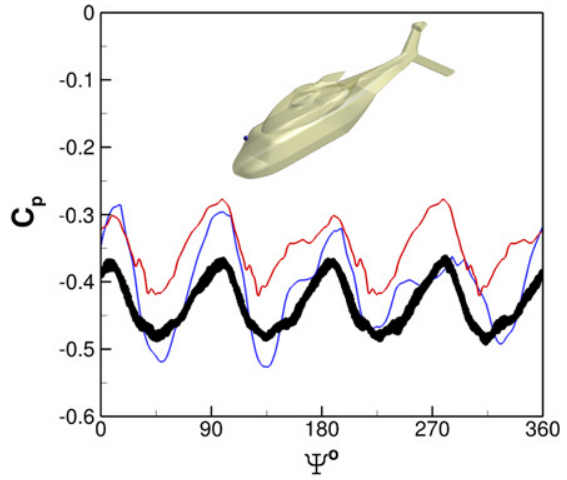

f)

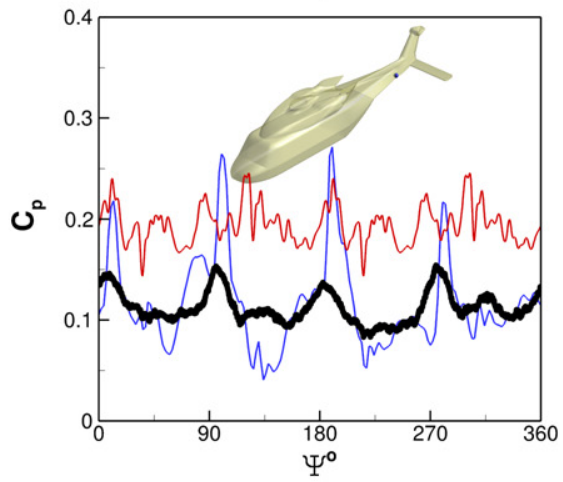

i)

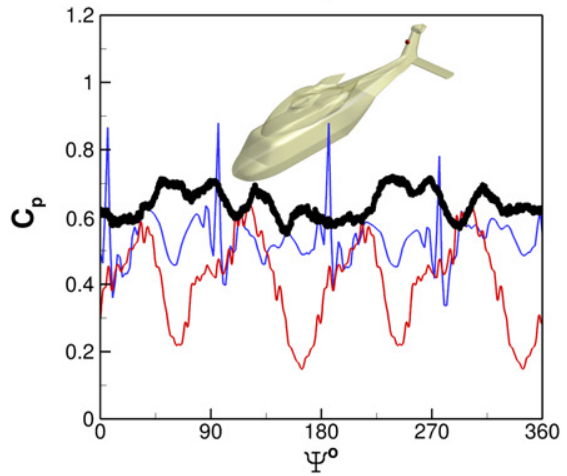

l)

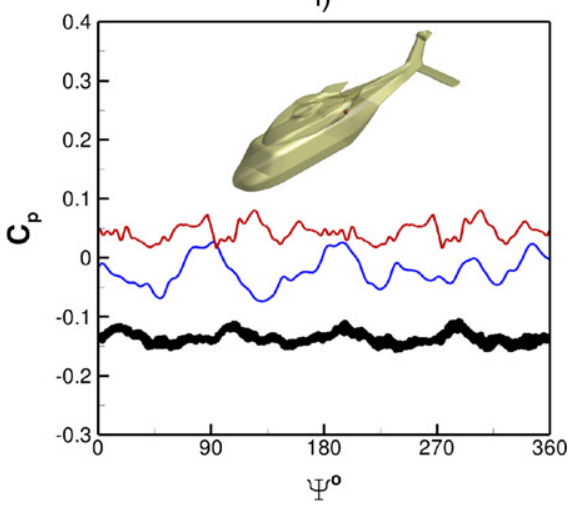

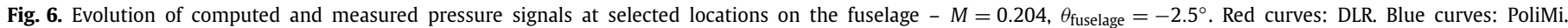
Symbols: experiment. (For interpretation of the references to color in this figure, the reader is referred to the web version of this article.)

show the pressure at selected radial positions: $r / R=0.500,0.825$ and 0.975 , for one main rotor revolution at azimuthal spacing of $30^{\circ}$, in order to represent all flow conditions encountered by the blade. The unsteady pressure sensors were distributed on three blades, but are gathered together to have a meaningful chordwise distribution. For each blade a different color is used in the fig- 

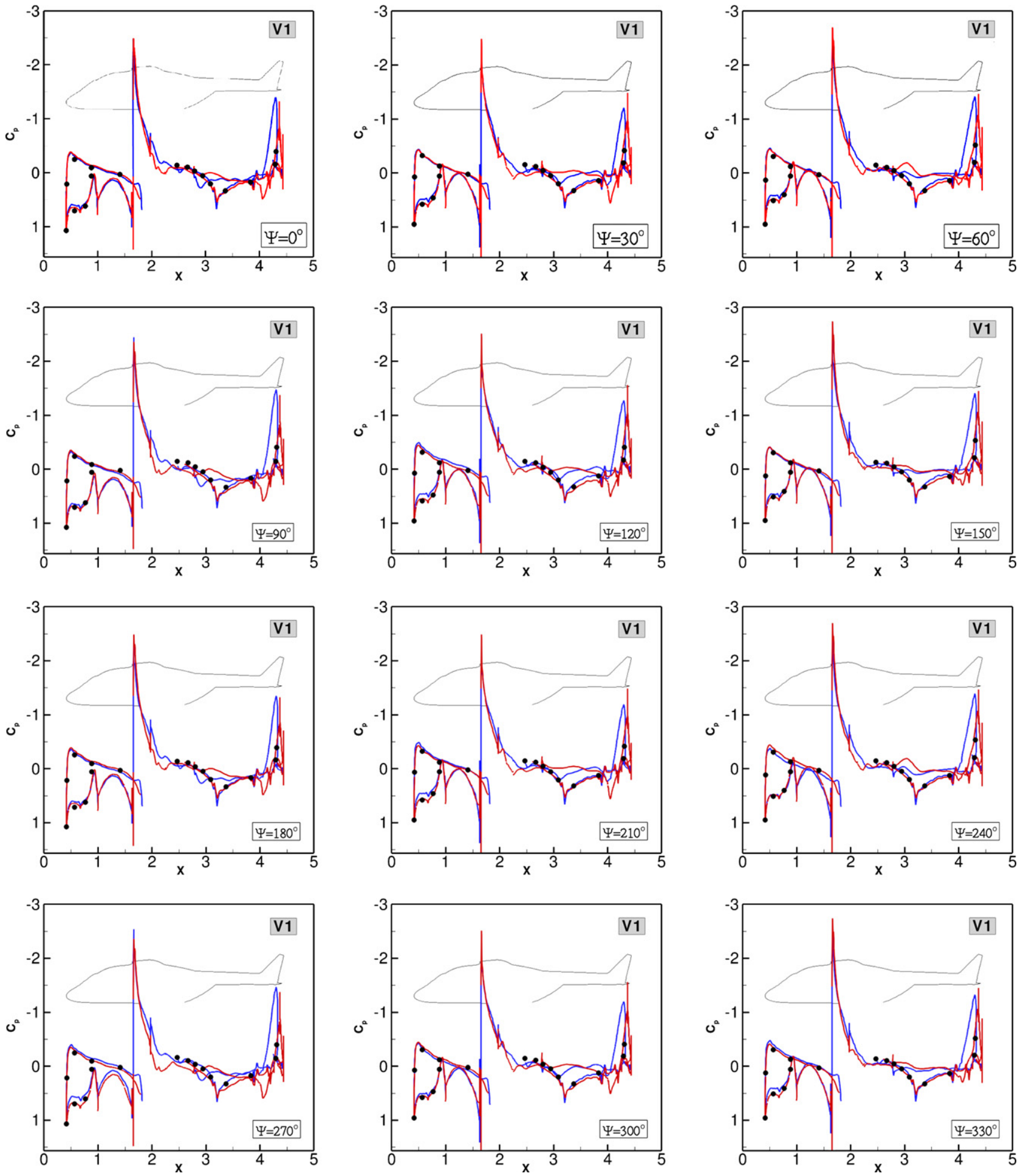

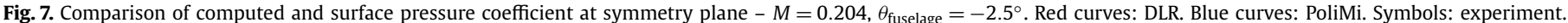
(For interpretation of the references to color in this figure, the reader is referred to the web version of this article.)

ures. The experimental data shown were averaged over 150 rotor revolutions, but in this case the differences with raw data are minimal.

Qualitatively, the computations captured the pressure pattern well over the whole revolution for the three radial locations. At the inboard radial station, PoliMi pressure values are generally higher than the DLR pressure on the suction side (Fig. 8). At $r / R=0.825$, Fig. 9, the computational results are very close to the experimental data. Apart from discrepancy on the suction side in the range
$\Psi=30^{\circ}$ to $90^{\circ}$, and at $\Psi=150^{\circ}$, the numerical results match the measurements very well. Similar good agreement is found in Fig. 10 for the radial location $r / R=0.975$. A reduction in the advancing range discrepancy found in Fig. 9 is observed.

The differences between DLR and PoliMi results diminish with radial distance, where instead the largest differences between the two modeling approaches should be expected. The elastic effects are therefore not large and were most probably compensated in PoliMi's calculations by a different blade kinematics, which leads 

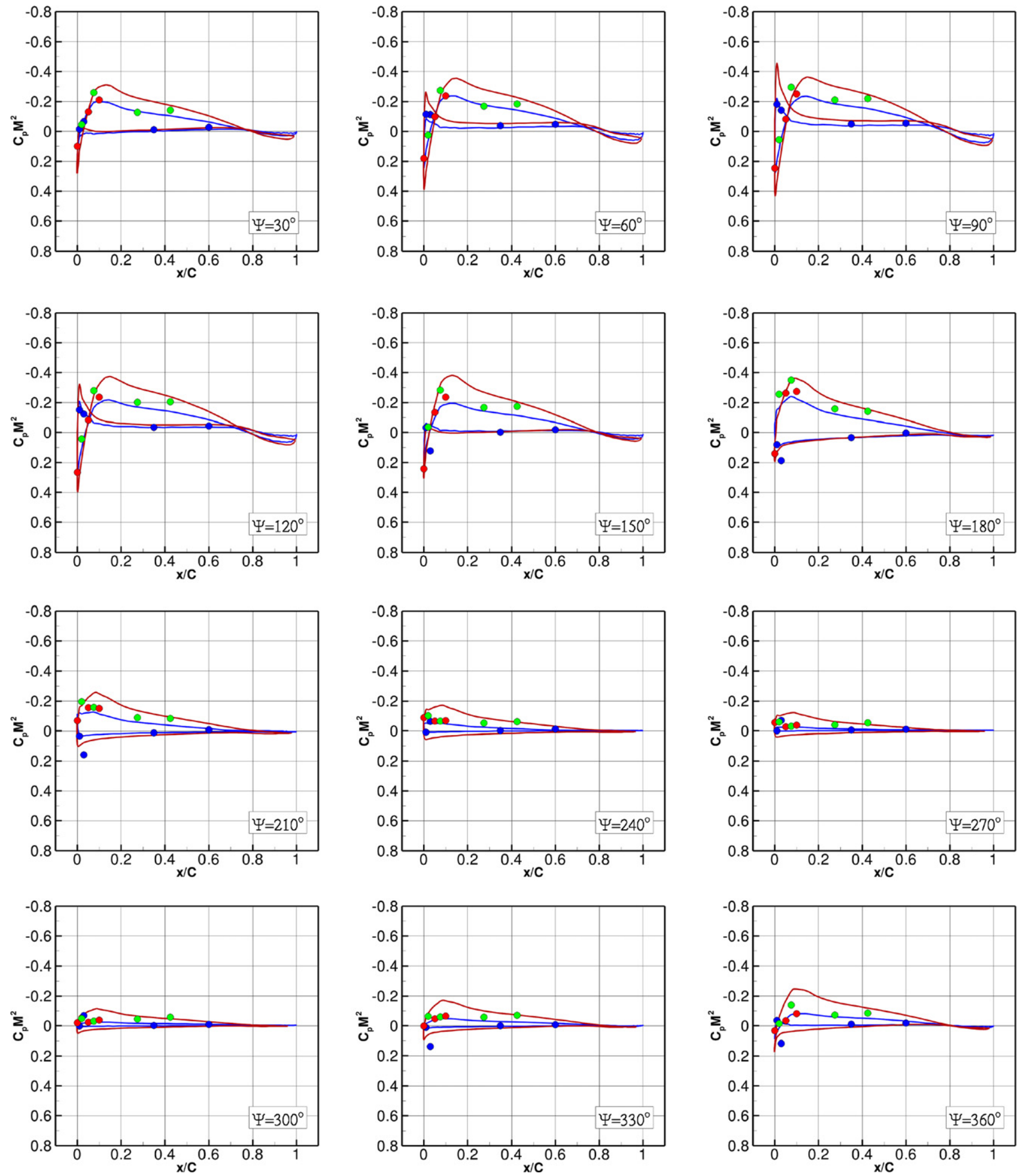

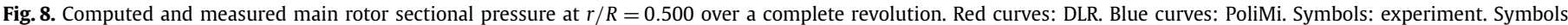
of different colors denote different blades. (For interpretation of the references to color in this figure, the reader is referred to the web version of this article.)

finally to a similar local effective angle of attack. Fig. 11 reports the normalized pitch and flap combinations for the two calculations. We recall that PoliMi simulations were run using the measured kinematics, while in DLR simulations the pitch and flap variations are obtained from the computed trim and blade dynamics. It is noticeable how the computed DLR pitch is rather similar with the experimental one, while somewhat larger differences can be observed in the flap motion.
The good agreement found for the unsteady pressure on the main rotor is confirmed plotting the sectional loads versus azimuth, see Fig. 12, where numerical and experimental results are reported. The numerical sectional loads, extracted from the computed pressure distributions, are not fully consistent with the experimental data, since during the integration all available pressure values are utilized and not only those gathered at the same chordwise locations than the experimental sensors. Despite some quan- 

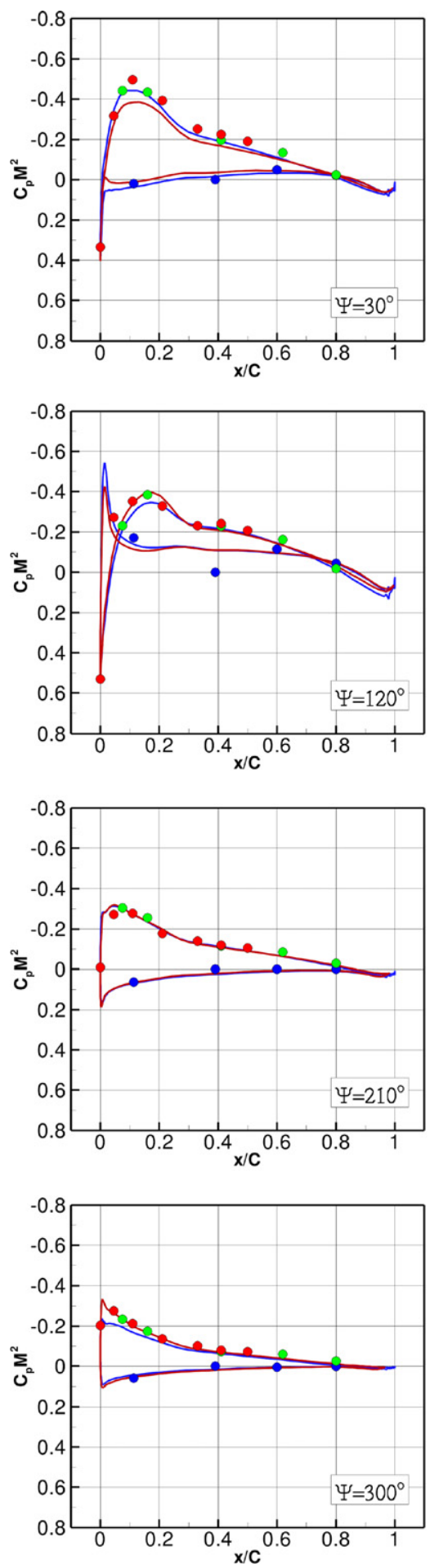
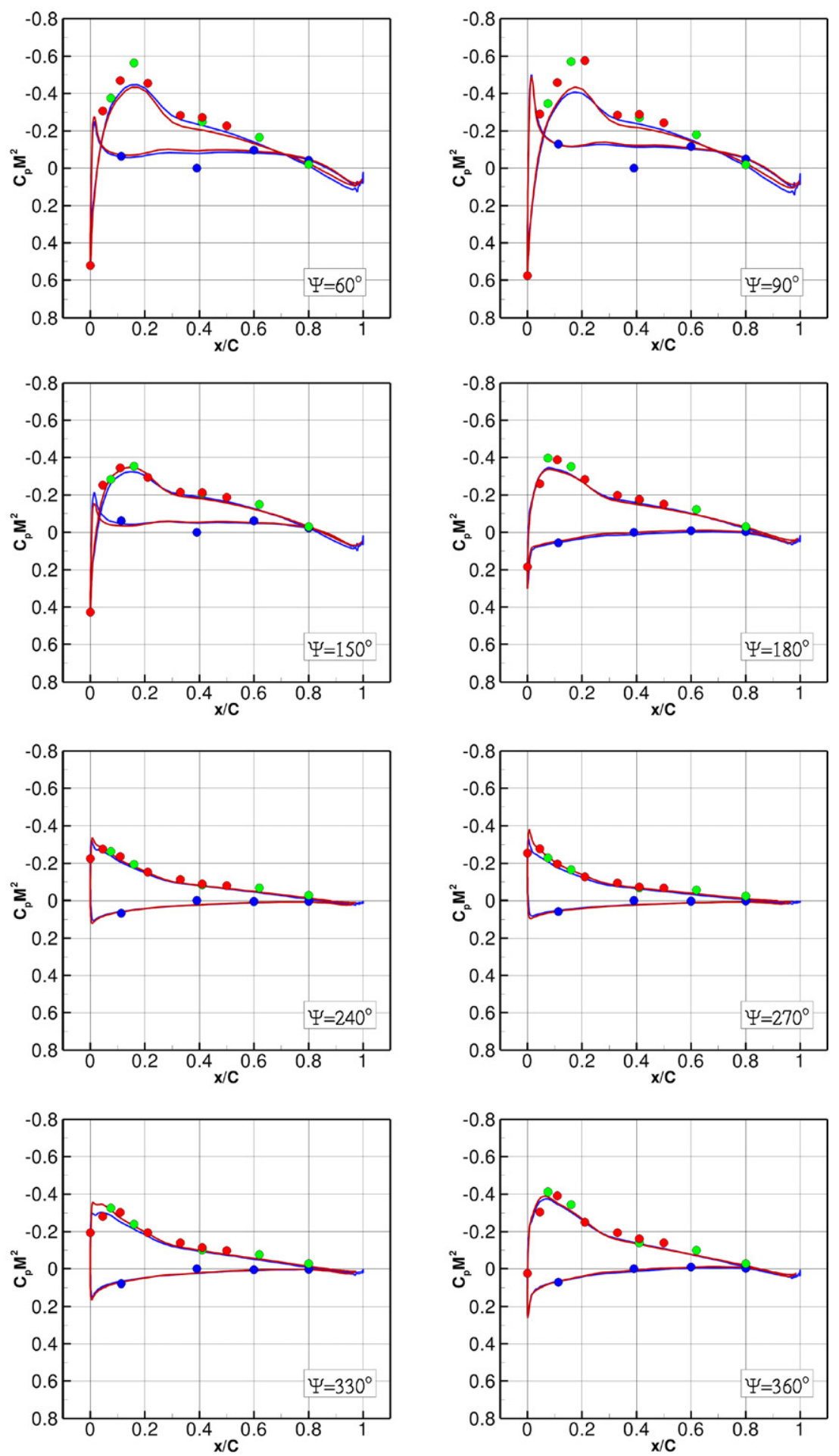

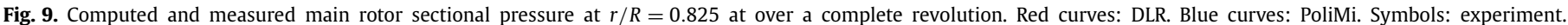
Symbols of different colors denote different blades. (For interpretation of the references to color in this figure, the reader is referred to the web version of this article.)

titative differences, the qualitative behavior is well represented by both codes during the whole blade revolution.

Tail rotor pressures at the radial locations $r / R=0.97$ are presented respectively in Fig. 13. Similar to the main rotor data, sectional pressure plots are shown with azimuthal spacing of $30^{\circ}$. DLR computations were performed using the experimental pitch values while the commands for the flap motion were taken from the blind test matrix. This obviously impaired the accuracy of predictions below the level of the blind test computations reported in [16]. On the other hand, PoliMi adopted the experimental values for both pitch and flap control angles, but this did not lead to a noticeable better agreement with the experimental data. It has to be noted that the tail rotor simulations were surely under-resolved in time, since the tail rotor has a fivefold rotational speed with respect to the main rotor.

\subsection{Flow field}

The complexity of the flow field around the complete helicopter can be appreciated from a qualitative visualization, using the Q- 

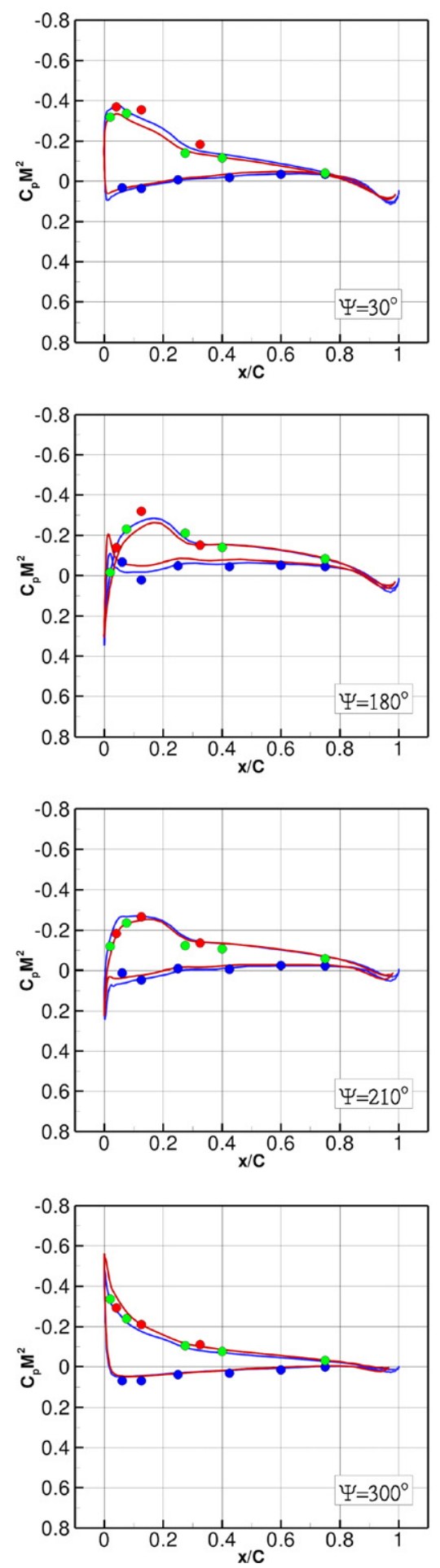
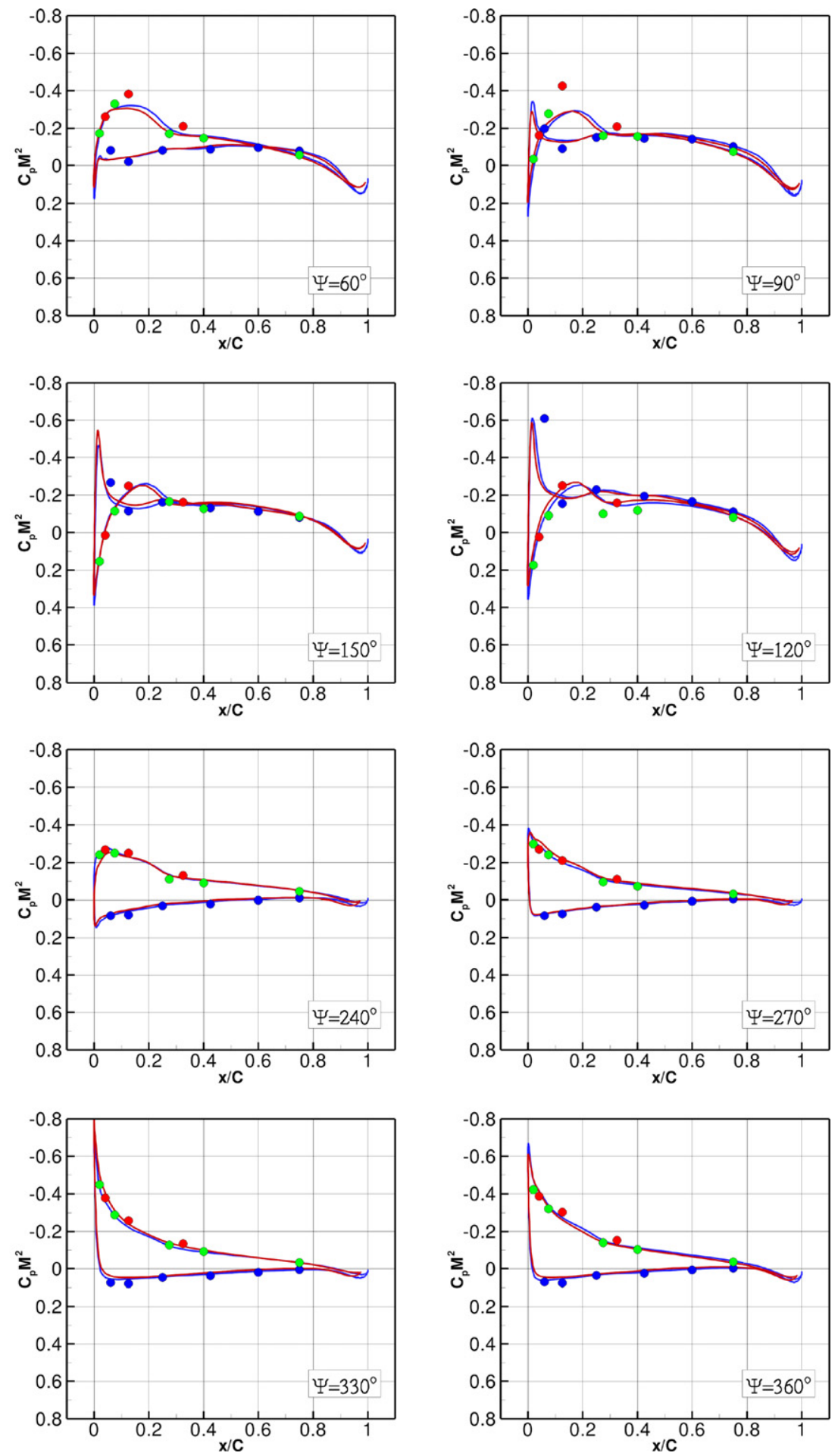

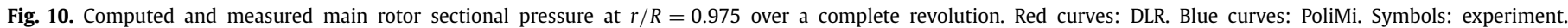
Symbols of different colors denote different blades. (For interpretation of the references to color in this figure, the reader is referred to the web version of this article.)

criterion [14], of the rotor and hub wakes as shown in Fig. 14. Note that the value of the iso-contour is optimized based on PoliMi results.

Three-dimensional velocity field PIV data were gathered during the experimental campaign [25] in several cross-planes, described in Fig. 15. The data were ensemble averaged at a given azimuth over 10 to 100 images, depending on the quality of the images themselves. The out-of-plane vorticity component was computed during post-processing. For the cruise test case here analyzed, only PIV1 (above the tail boom) and PIV2 (behind the back door) planes were considered, at different $\mathrm{X}$ distances along the fuselage axis.

To analyze the hub wake, we will consider a PIV1 plane approximately 1 meter behind the rotor hub. Unfortunately, PoliMi results are not available at the same azimuthal angles at which experimental data are provided; therefore we will first compare DLR results with experiments and then with PoliMi computations at a nearby azimuth. Figs. 16 and 17 plot the out-of-plane component 


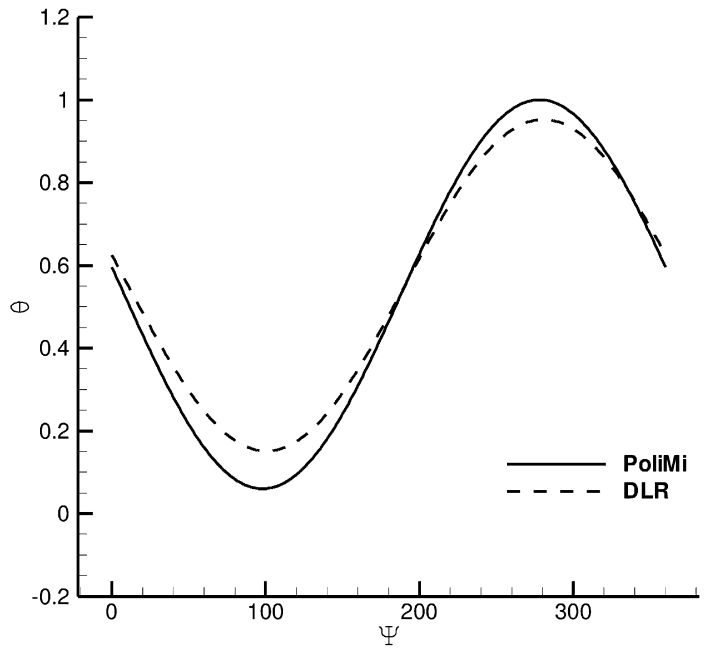

a) Normalized pitch

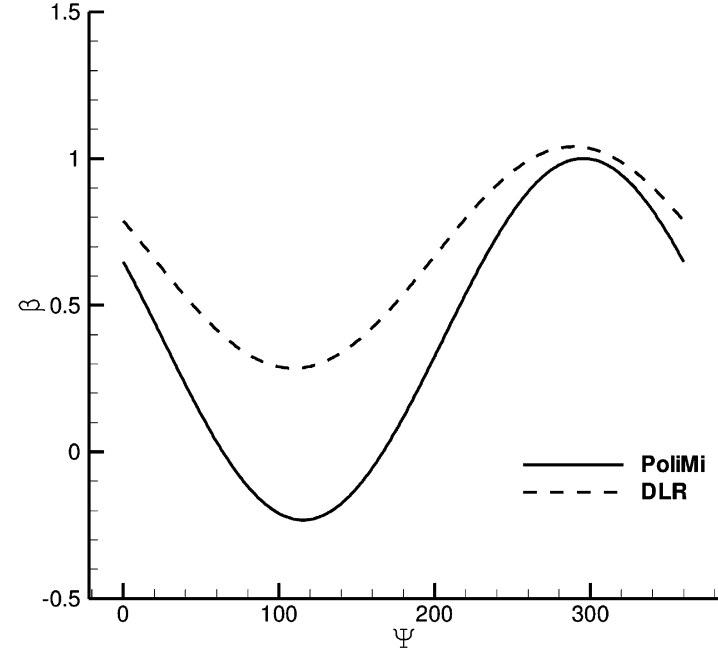

b) Normalized flap

Fig. 11. Normalized pitch and flap angle variation over one revolution. Solid line: PoliMi. Dotted line: DLR.

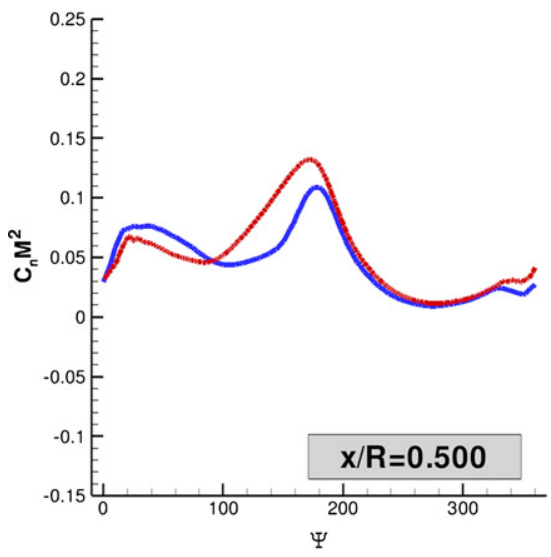

Complete helicotper: $\mathrm{M}=0.204, \theta=-2.5^{\circ}$
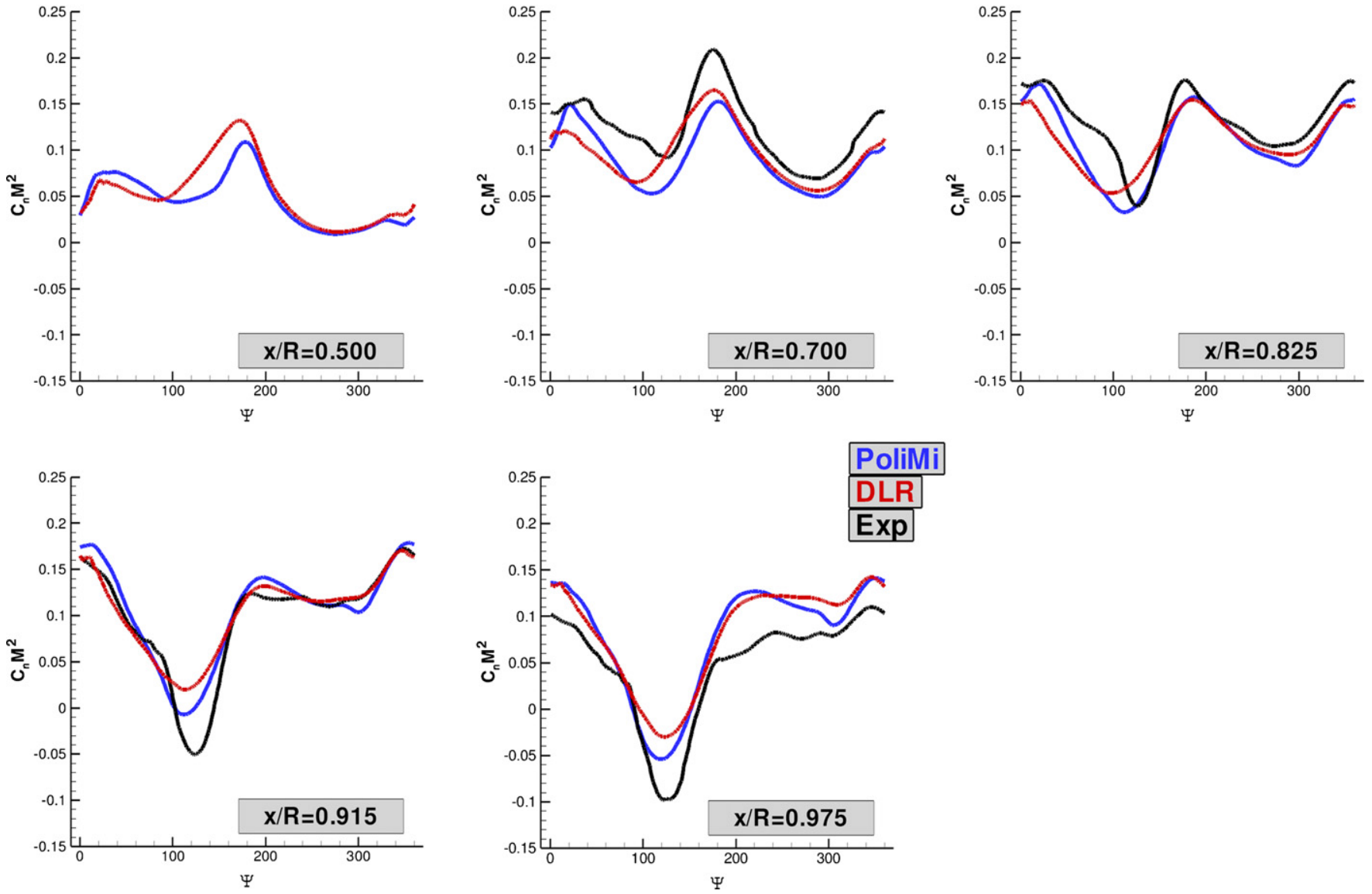

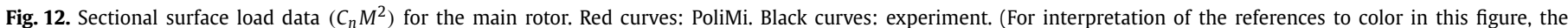
reader is referred to the web version of this article.)

of the vorticity and velocity vectors at $\Psi=22.5^{\circ}$, together with pseudo-stream traces. The structure of the hub wake predicted by DLR computations looks more asymmetric than the experimental one: since the measured data are ensemble averaged, this seems to imply a strong unsteadiness of the wake itself. It has to be noticed that the trace of the passing blade wake, clearly seen in the upper part of Fig. 16, is well resolved by the computations. A code-to-code comparison at $\Psi=30^{\circ}$ is carried out in Figs. 18 and 19. Looking at the DLR velocity results at the two azimuthal values (Figs. 17 and 19), it can be stated that the computed flow is evolving smoothly. Both codes manage to capture the passing blade wake and the asymmetry of the hub wake, but show also 

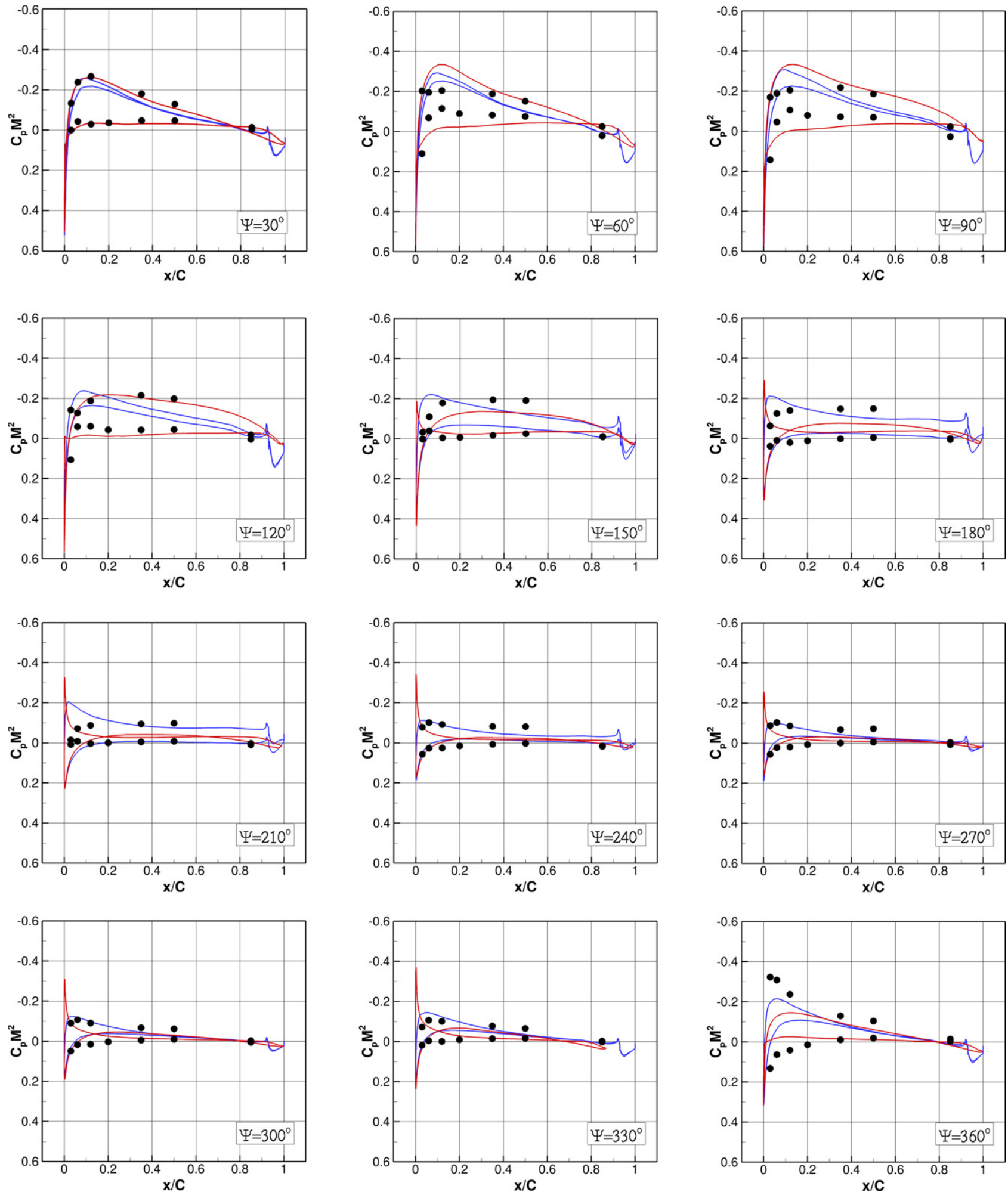

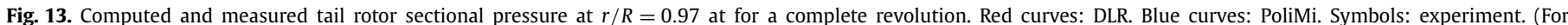
interpretation of the references to color in this figure, the reader is referred to the web version of this article.)

noticeable differences in the flow field. It has to be observed that the vorticity plots are affected by the difficulty of the plotting program to deal with a region of overlapping meshes. The PoliMi grids depicted on the right side of Fig. 18 are an example of how the blade grid is blanking out the fuselage grid in the overlapping region.
Finally, we consider two PIV2 cross-sectional planes behind the fuselage back door, at distances respectively of 1.16 and 1.46 meters from the hub. Plots in Figs. 20 and 21 show again the outof-plane velocity component together with pseudo-stream traces. The wake here is characterized by the presence of two well organized counter-rotating vortices that are fairly well resolved by 

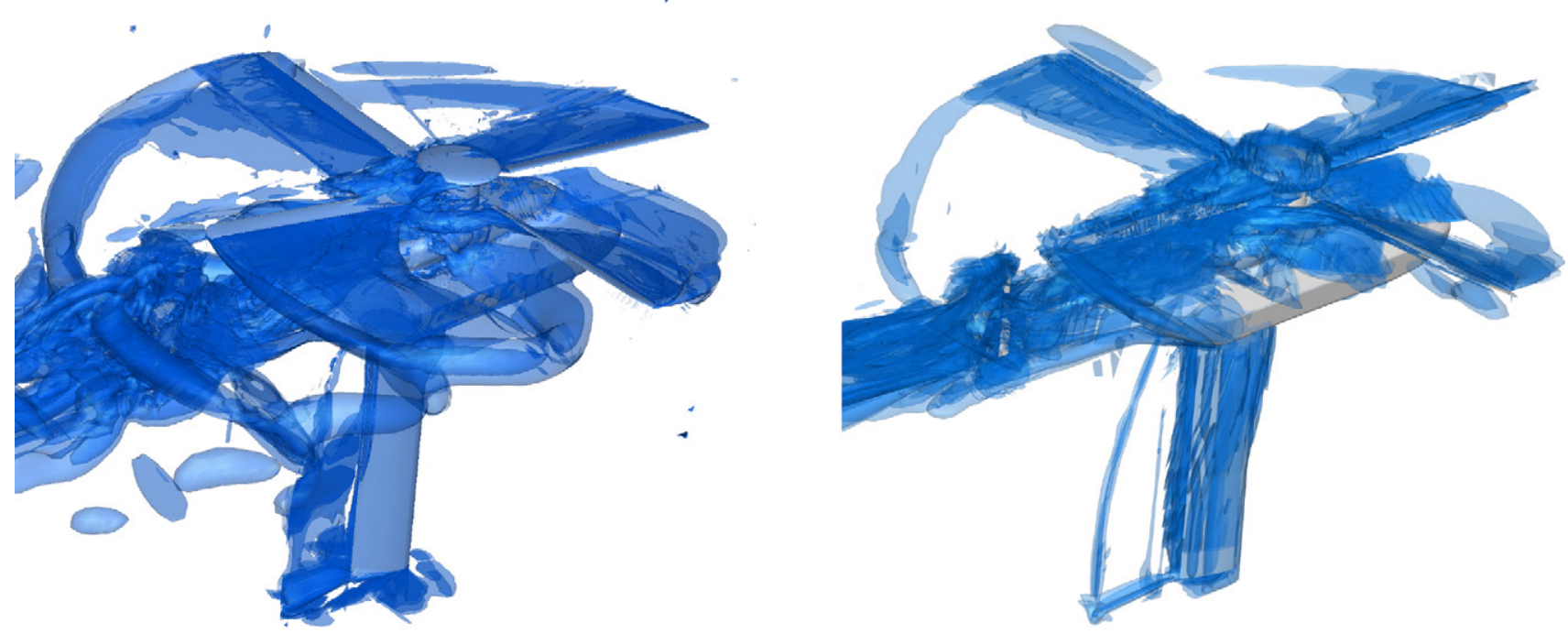

Fig. 14. Wake visualization with Q-criterion. Left: PoliMi results. Right: DLR results.

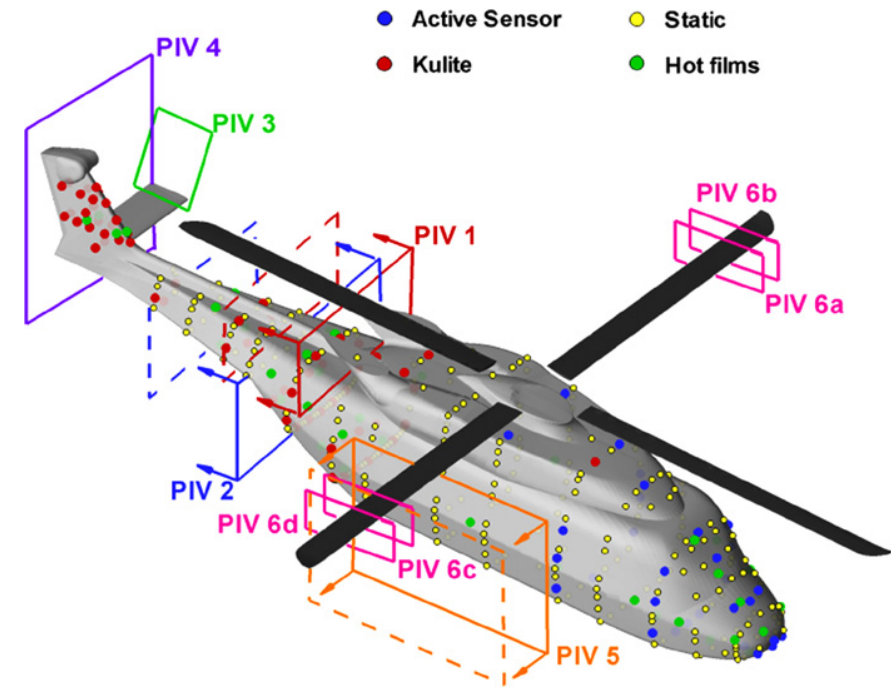

Fig. 15. Steady and unsteady pressure sensors locations on the fuselage and PIV measurement position.

the numerical simulations, although quantitative differences remain.

\section{Conclusions}

Results of the simulations of a complete helicopter flow filed, performed by DLR and PoliMi within the GOAHEAD project, were examined by comparison with experimental data. The comparison means to assess the capability of present CFD codes in predicting the aerodynamic loading of a full helicopter configuration and to ascertain the importance of elastic effects on the predictions, since different models were utilized: weak fluid-structure coupling was iteratively applied by DLR to trim the main rotor while PoliMi applied the experimental rotor controls with rigid blades.

Good agreement between computed and measured pressure signals on the front upper part of the fuselage in terms of phase and magnitude could be found. On the tail boom, tail fins and fuselage back door a less satisfactory comparison was observed. Both CFD approaches predicted fuselage surface pressure with similar accuracy, indicating a negligible influence of the modeling approach on the fuselage loads.

Some discrepancy between DLR and PoliMi was found on the chordwise pressure distribution at the inboard stations of the main rotor blades. However, the observed differences decreased rapidly in the direction of the blade tip. Furthermore, the agreement of sectional loads with experimental data is rather satisfactory.

The lack of accurate description of the tail rotor motion resulted in an evident mismatch between the CFD results and the experimental data.

Fluid-structure-flight mechanics coupling is an essential approach for accurate prediction of main rotor power. Coupled simulation predicted the power with an accuracy of $0.3 \%$, while rigid blade assumption predicted $15 \%$ less power.

\section{Acknowledgements}

This investigation was carried out as a part of the European Union Specific Targeted Research Project GOAHEAD (GROWTH Contract Number AST4-CT-2005-516074). The authors would like to thank the European Union for financial support.
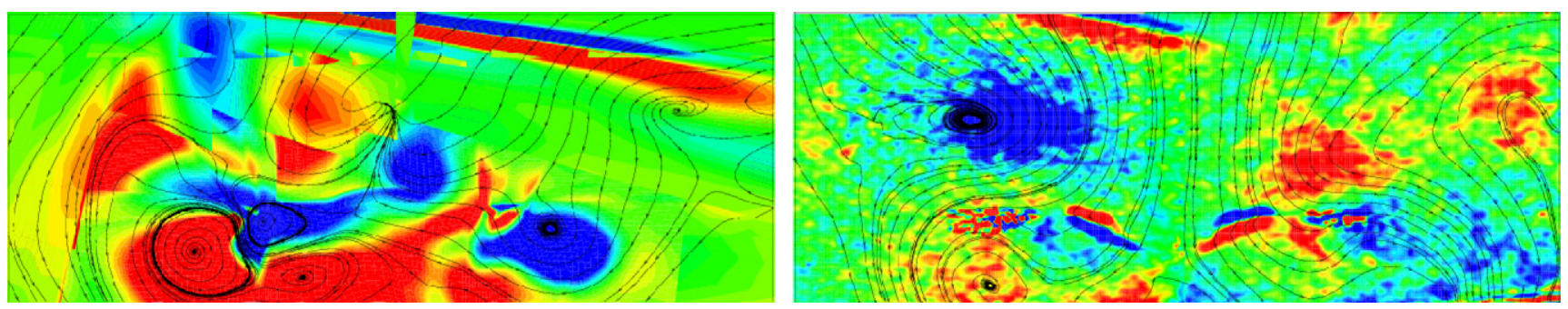

Fig. 16. Out-of-plane vorticity component and pseudo-stream traces in a PIV1 plane at $X=0.970 \mathrm{~m}, \psi=22.5^{\circ}$ : DLR results (left), experiments (right). 

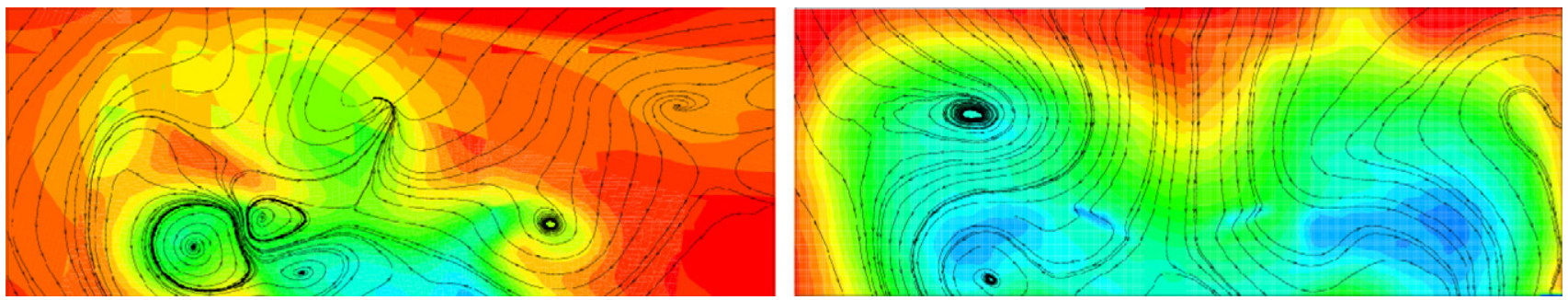

Fig. 17. Out-of-plane velocity component and pseudo-stream traces in a PIV1 plane at $X=0.970 \mathrm{~m}, \psi=22.5^{\circ}$ : DLR results (left), experiments (right).
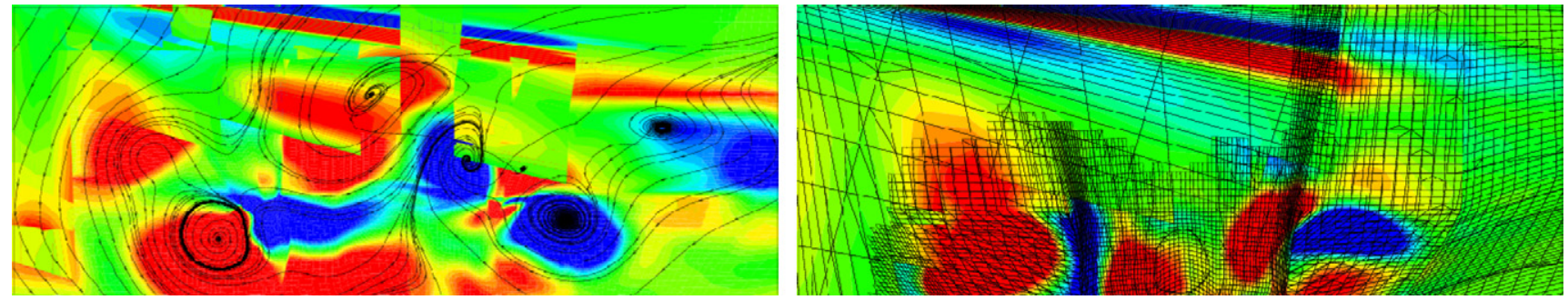

Fig. 18. Out-of-plane vorticity component in a PIV1 plane at $X=0.970 \mathrm{~m}, \psi=30^{\circ}$ : DLR results (left), PoliMi results (right).
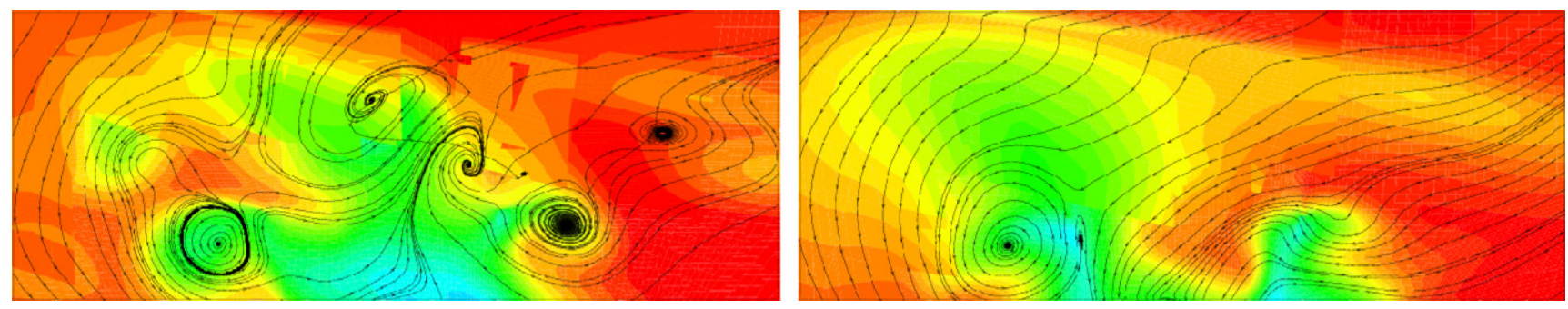

Fig. 19. Out-of-plane velocity component and pseudo-stream traces in a PIV1 plane at $X=0.970 \mathrm{~m}, \psi=30^{\circ}$ : DLR results (left), PoliMi results (right).
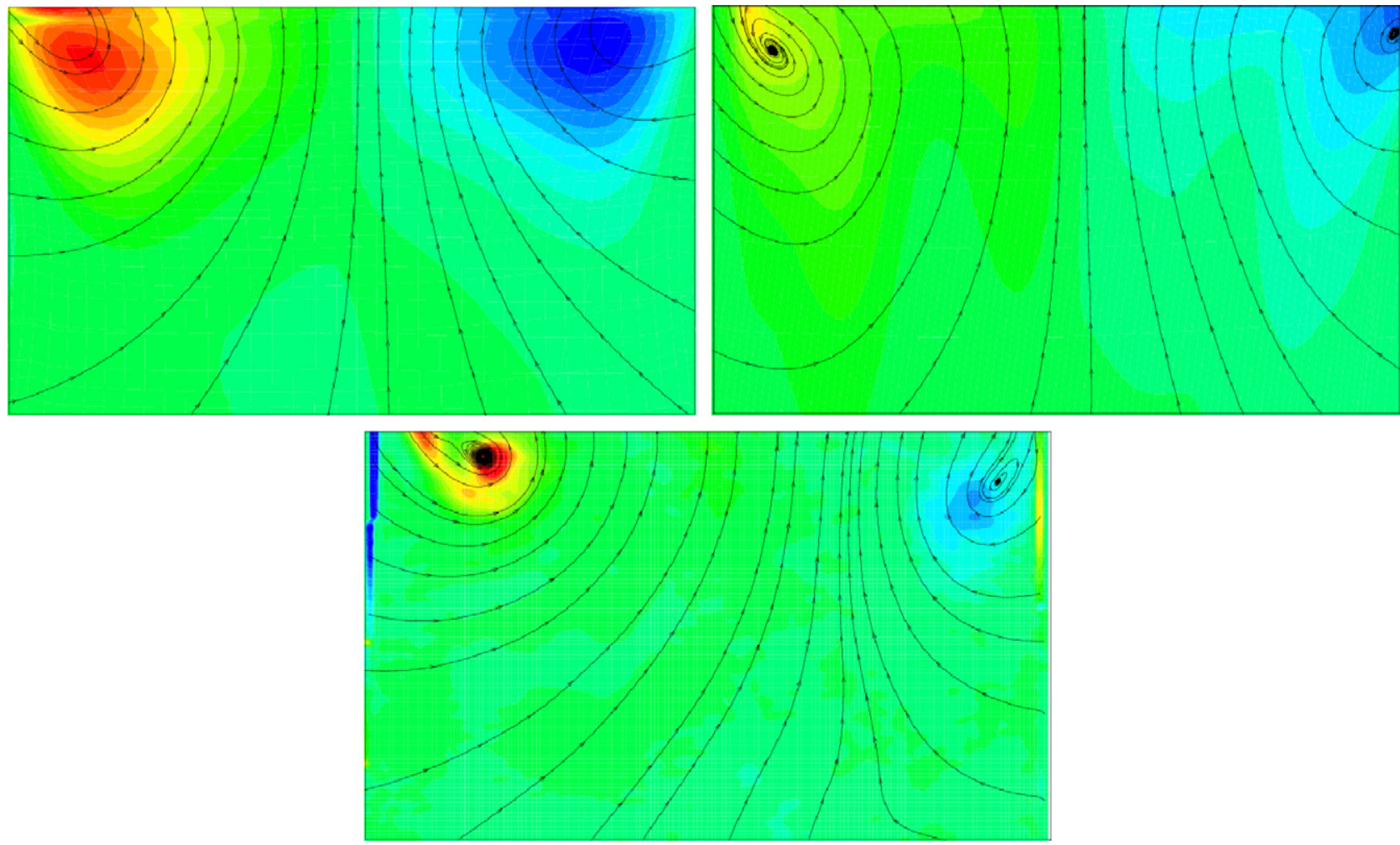

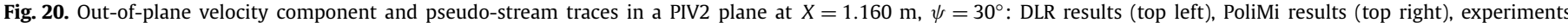
(bottom). 

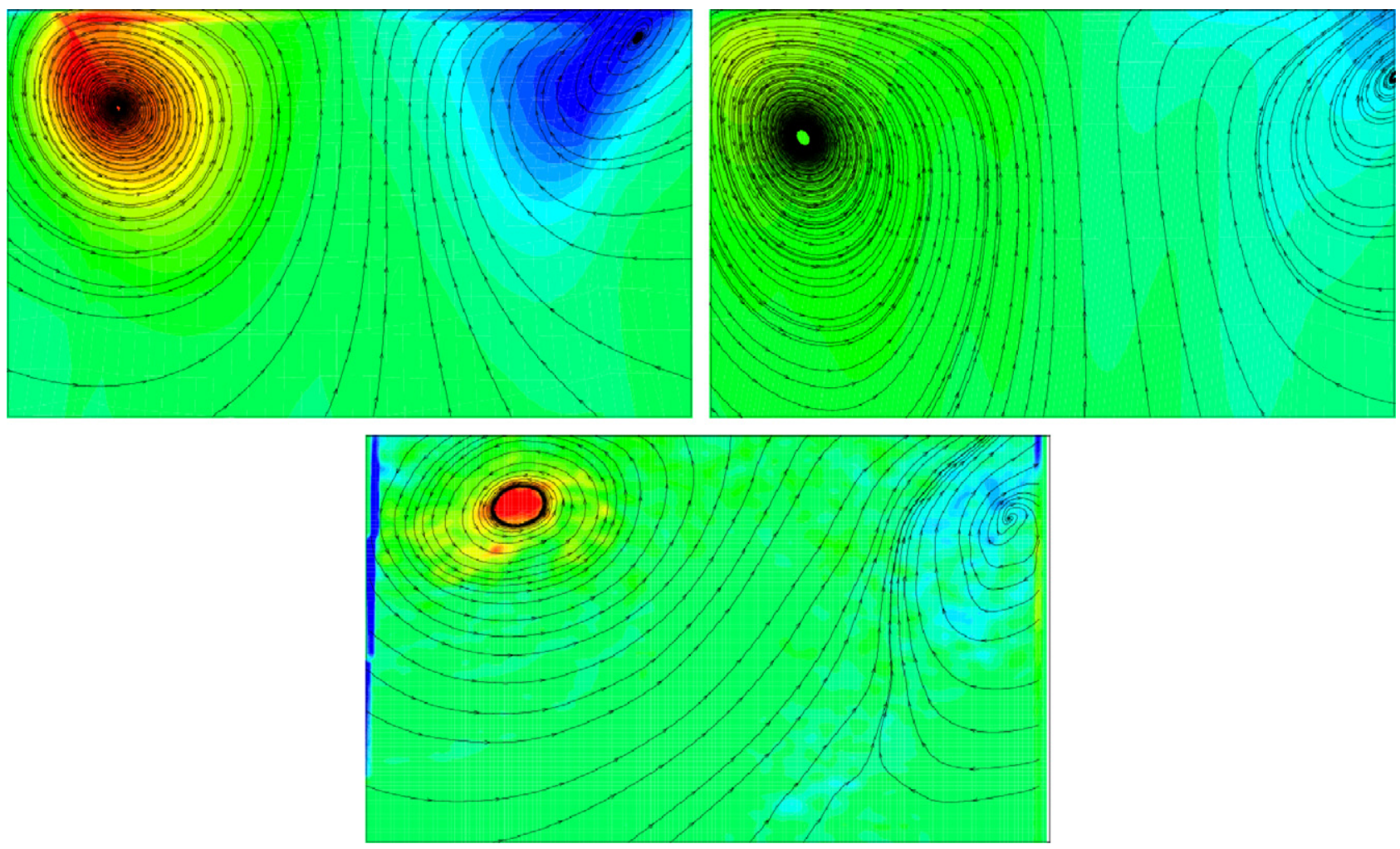

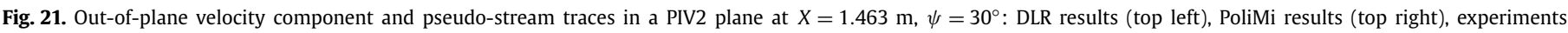
(bottom).

\section{References}

[1] J.A. Benek, P.G. Buning, J.L. Steger, A 3-D chimera grid embedding technique, in: 7th Computational Fluid Dynamics Conference, Cincinnati, OH, AIAA-19851523, 1985.

[2] J.A. Benek, J.L. Steger, F.C. Doughertz, A flexible grid embedding technique with application to the Euler equations, AIAA paper 83-1944, 1983.

[3] B. Benoit, A.-M. Dequin, K. Kampa, W. von Grünhagen, P.-M. Basset, B. Gimonet, HOST: a general helicopter simulation tool for Germany and France, in: 56th Annual Forum of the American Helicopter Society, Virginia Beach, VA, 2000.

[4] M. Bhagwat, A. Dimanlig, H. Saberi, E. Meadwcroft, B. Panda, R. Strawn, CFD/CSD coupled trim solution for the dual-rotor $\mathrm{CH}-47$ helicopter including fuselage modeling, in: American Helicopter Society Specialists' Conference on Aeromechanics, San Francisco, CA, 2008.

[5] M. Biava, A. Pisoni, A. Saporiti, L. Vigevano, Efficient rotor aerodynamics predictions with an Euler method, in: Proceedings of the 29th European Rotorcraft Forum, Friedrichshafen, Germany, 2003.

[6] O.J. Boelens, G. Barakos, M. Biava, A. Brocklehurst, M. Costes, A. D’Alascio, M Dietz, D. Drikakis, J. Ekaterinaris, I. Humby, W. Khier, B. Knutzen, J. Kok, F. LeChuiton, K. Pahlke, T. Renaud, T. Schwarz, R. Steijl, L. Sudre, H. van der Ven, L. Vigevano, B. Zhong, The blind-test activity of the GOAHEAD project, in: Proceedings of the 33rd European Rotorcraft Forum, Kazan, Russia, 2007.

[7] M. Chaffin, J. Berry, Navier-Stokes and potential theory solutions for a helicopter fuselage and comparison with experiments, NASA TM-4566, 1994.

[8] W.M. Chan, P.G. Buning, Zipper grids for force and moment computation on overset grids, AIAA Paper 95-1681, 1995.

[9] G. Chesshire, W.D. Henshaw, Composite overlapping meshes for the solution of partial differential equations, J. Comp. Phys. 90 (1990) 1-64.

[10] M. Dietz, Simulation der Umströmung von Hubschrauberkonfigurationen unter Berück-sichtigung von Strömung-Struktur-Kopplung und Trimmung, Ph.D. Thesis, Institut für Aerodynamik und Gasdynamik, Universität Stuttgart, Stuttgart, Germany, 2009.

[11] M. Dietz, M. Keßler, E. Krämer, Trimmed Simulation of a Complete Helicopter Configuration Using Fluid-Structure Coupling, High Performance Computing in Science and Engineering, Springer Verlag, 2007.

[12] M. Dietz, W. Khier, B. Knutzen, S. Wagner, E. Krämer, Numerical simulation of a full helicopter configuration using weak fluid-structure coupling, in: 46th AIAA Aerospace Science Meeting, Reno, NV, AIAA-2008-01-07, 2008.

[13] M. Dietz, E. Krämer, S. Wagner, A. Altmikus, Weak coupling for active advanced rotors, in: Proceedings of the 31st European Rotorcraft Forum, Florence, Italy, September 2005.
[14] J.C.R. Hunt, A. Wray, P. Moin, Eddies, stream, and convergence zones in turbulent flows, Center for Turbulence Research Report CTR-S88, 1988.

[15] A. Kenyon, R. Brown, Wake dynamics and rotor-fuselage aerodynamic interactions, J. Amer. Helicopter Soc. 54 (1) (2009) 012003/1-012003/18.

[16] W. Khier, Numerical simulation of air flow past a full helicopter configuration, in: Proceedings of the 35th European Rotorcraft Forum, Hamburg, Germany, 2009.

[17] W. Khier, M. Dietz, T. Schwarz, S. Wagner, Trimmed CFD simulation of a complete helicopter configuration, in: Proceedings of the 33rd European Rotorcraft Forum, Kazan, Russia, 2007.

[18] W. Khier, T. Schwarz, J. Raddatz, Time-accurate simulation of the flow around the complete BO105 wind-tunnel model, in: Proceedings of the 31st European Rotorcraft Forum, Florence, Italy, 2005.

[19] J. Kim, N. Komerath, Summary of the interaction of a rotor wake with a circular cylinder, AIAA J. 33 (3) (1995) 470-478.

[20] N. Kroll, B. Eisfeld, H.M. Bleecke, The Navier-Stokes code FLOWer, in: Notes on Numerical Fluid Mechanics, vol. 71, Vieweg, Braunschweig, 1999, pp. 58-71.

[21] N. Kroll, C.-C. Rossow, K. Becker, F. Thiele, The MEGAFLOW project, Aerospace Science and Technology 4 (2000) 223-237.

[22] K. Pahlke, The GOAHEAD project, in: Proceedings of the 33rd European Rotorcraft Forum, Kazan, Russia, 2007.

[23] K. Pahlke, B. Van der Wall, Calculation of multi-bladed rotors in high speed forward flight, in: Proceedings of the 27th European Rotorcraft Forum, Moscow, Russia, 2001.

[24] M. Potsdam, M. Smith, T. Renaud, Unsteady computations of rotor-fuselage interaction, in: Proceedings of the 35th European Rotorcraft Forum, Hamburg, Germany, 2009.

[25] M. Raffel, F. De Gregorio, W. Sheng, G. Gibertini, A. Seraudie, K. De Groot, B.G. van der Wall, Generation of an advanced helicopter experimental aerodynamic database, in: Proceedings of the 35th European Rotorcraft Forum, Hamburg, Germany, 2009.

[26] T. Renaud, A. Le Pape, C. Benoit, Unsteady Euler and Navier-Stokes computations of a complete helicopter, in: Proceedings of the 31st European Rotorcraft Forum, Florence, Italy, 2005.

[27] T. Renaud, D. O'Brien, M. Smith, M. Potsdam, Evaluation of isolated fuselage and rotor-fuselage interaction using CFD, in: 60th Annual Forum of the American Helicopter Society, Baltimore, MD, 2004.

[28] R. Rudnik, Untersuchung der Leistungsfähigkeit von ZweigleichungsTurbulenzmodellen bei Profilumströmungen, Deutsches Zentrum für Luftund Raumfahrt e.V., FB 97-49, 1997.

[29] T. Schwarz, Development of a wall treatment for Navier-Stokes computations using the overset-grid technique, in: Proceedings of the 26th European Rotorcraft Forum, The Hague, The Netherlands, 2000. 
[30] J. Sidès, K. Pahlke, M. Costes, Numerical simulation of flows around helicopters at DLR and ONERA, Aerospace Science and Technology 5 (2001) 35-53.

[31] P.R. Spalart, S.R. Allmaras, A one-equation turbulence model for aerodynamic flows, AIAA paper 92-439, 1992.

[32] R. Steijl, G. Barakos, Sliding mesh algorithm for CFD analysis of helicopter rotor-fuselage aerodynamics, Int. J. Num. Meth. in Fluids 58 (2008) 527-549.
[33] R. Steijl, G. Barakos, Computational study of helicopter rotor-fuselage aerodynamic interactions, AIAA J. 47 (9) (2009) 2143-2157.

[34] V. Venkatakrishnan, On the accuracy of limiters and convergence to steady state solutions, AIAA paper 93-0880, 1993.

[35] D.C. Wilcox, Reassessment of the scale-determining equation for advanced turbulence models, AIAA J. (1988) 1299-1310. 